\title{
Diisonitrile-Mediated Reactive Oxygen Species Accumulation Leads to Bacterial
}

\section{Growth Inhibition}

Mengyi Zhu, Lijuan Wang, Wei Zhang, Zhiwen Liu, Muhammad Ali, Muhammad

Imtiaz, and Jing $\mathrm{He}^{*}$

*Corresponding author:

hejingji@mail.hzau.edu.cn

\section{Supporting Information}

\section{Table of contents}

Table S1. ${ }^{1} \mathrm{H}$ and ${ }^{13} \mathrm{C}$ NMR data of compound 9, 11, and 12.

Table S2. ${ }^{1} \mathrm{H}(600 \mathrm{MHz})$ and ${ }^{13} \mathrm{C}(150 \mathrm{MHz})$ NMR Data of the pyran ring of SF2768 and its analogues.

Table S3. The detailed data of the ICP-MS analysis.

Figure S1. (A) Detection of SF2768 analogues in the fermentation broth of

S. lividans::p13C. (B) HR-ESI-MS analysis of compound 9, 11 and 12.

Figure S2. Detection of the anomers of SF2768 on HPLC.

Figure S3. The anomeric protons of SF2768 (1) and its analogues.

Figure S4. Time-course of the ROS accumulation in eight bacteria.

Figure S5. NMR spectra of 9 in DMSO- $d_{6}$ at $600 \mathrm{MHz}$ and $151 \mathrm{MHz}$.

Figure S6. NMR spectra of 11 in DMSO- $d_{6}$ at $600 \mathrm{MHz}$ and $151 \mathrm{MHz}$.

Figure S7. NMR spectra of 12 in DMSO- $d_{6}$ at $600 \mathrm{MHz}$ and $151 \mathrm{MHz}$.

\section{Supplemental References}

(1) Amano, S. I.; Sakurai, T.; Endo, K.; Takano, H.; Beppu, T.; Furihata, K.; Sakuda, S.; Ueda, K. J. Antibiot. 2011, $64,703$.

(2) Wang, L.; Zhu, M.; Zhang, Q.; Zhang, X.; Yang, P.; Liu, Z.; Deng, Y.; Zhu, Y.; Huang, X.; Han, L.; Li, S.; He, J. ACS Chem. Biol. 2017, 12, 3067-3075.

(3) Zhu, M.; Wang, L.; Zhang, Q.; Ali, M.; Zhu, S.; Yu, P.; Gu, X.; Zhang, H.; Zhu, Y.; He, J. Org. Lett. 2018, 20, 3562-3565.

(4) Zhu, M.; Wang, L.; He, J. ACS Chem. Biol. 2019, 14, 256-265. 
Table S1. ${ }^{1} \mathrm{H}$ and ${ }^{13} \mathrm{C}$ NMR data $(600 \mathrm{MHz}, 151 \mathrm{MHz})$ for compound 9, 11, and 12.

Compound 9 (DMSO- $\left.d_{6}\right)$

\begin{tabular}{|c|c|c|c|c|c|}
\hline position & $\delta_{\mathrm{C}}$ & type & $\delta_{\mathrm{H}}(J$ in $\mathrm{Hz})$ & ${ }^{1} \mathrm{H}-{ }^{1} \mathrm{H}$ COSY & HMBC \\
\hline 1 & 20.29 & $\mathrm{CH}_{3}$ & $1.05, \mathrm{~d}(6.8)$ & $\mathrm{H}-8$ & $\mathrm{C}-8$ \\
\hline 2 & 20.43 & $\mathrm{CH}_{3}$ & $1.05, \mathrm{~d}(6.8)$ & $\mathrm{H}-9$ & C-9 \\
\hline 3 & 21.12 & $\mathrm{CH}_{3}$ & $1.99, \mathrm{~s}$ & & C-18 \\
\hline 4 & 23.19 & $\mathrm{CH}_{2}$ & $1.24, \mathrm{o} ; 1.17, \mathrm{o}$ & & \\
\hline 5 & 29.30 & $\mathrm{CH}_{2}$ & $1.35, \mathrm{o}$ & $\mathrm{H}-7$ & \\
\hline 6 & 30.65 & $\mathrm{CH}_{2}$ & $1.42, \mathrm{o} ; 1.31, \mathrm{o}$ & $\mathrm{H}-12$ & \\
\hline 7 & 38.70 & $\mathrm{CH}_{2}$ & $3.00, \mathrm{~m}$ & $\mathrm{H}-5, \mathrm{H}-20$ & C-4, C-5 \\
\hline 8 & 41.48 & $\mathrm{CH}$ & $4.13, \mathrm{~m}$ & H-1, H-10, H-21 & C-14, C-17 \\
\hline 9 & 41.56 & $\mathrm{CH}$ & $4.13, \mathrm{~m}$ & H-2, H-11, H-22 & $\mathrm{C}-15$ \\
\hline 10 & 42.40 & $\mathrm{CH}_{2}$ & $2.28, \mathrm{~m}$ & $\mathrm{H}-8$ & $\mathrm{C}-1, \mathrm{C}-8, \mathrm{C}-17$ \\
\hline 11 & 42.54 & $\mathrm{CH}_{2}$ & $2.14, \mathrm{~m}$ & $\mathrm{H}-9$ & C-2, C-9, C-16 \\
\hline 12 & 47.61 & $\mathrm{CH}$ & $3.91, \mathrm{~m}$ & H-19 & \\
\hline 13 & 65.97 & $\mathrm{CH}_{2}$ & $\begin{array}{l}\text { 3.98, dd }(10.8,6.6) \\
3.82 \text {, dd }(10.8,6.6)\end{array}$ & & $C-6, C-12, C-18$ \\
\hline 14 & 160.62 & $\mathrm{CH}$ & 7.91, o & & $\mathrm{C}-8$ \\
\hline 15 & 160.62 & $\mathrm{CH}$ & 7.91, o & & C-9 \\
\hline 16 & 170.03 & $\mathrm{C}$ & & & \\
\hline 17 & 170.19 & $\mathrm{C}$ & & & \\
\hline 18 & 170.76 & $\mathrm{C}$ & & & \\
\hline 19 & & $\mathrm{NH}$ & 7.78, d (8.4) & $\mathrm{H}-12$ & $\mathrm{C}-17$ \\
\hline 20 & & $\mathrm{NH}$ & $7.85, \mathrm{t}(5.6)$ & $\mathrm{H}-7$ & C-7, C-16 \\
\hline 21 & & $\mathrm{NH}$ & 7.96, o & $\mathrm{H}-8$ & $\mathrm{C}-14$ \\
\hline 22 & & $\mathrm{NH}$ & $7.96, \mathrm{o}$ & H-9 & C-15 \\
\hline
\end{tabular}

*o: overlapped

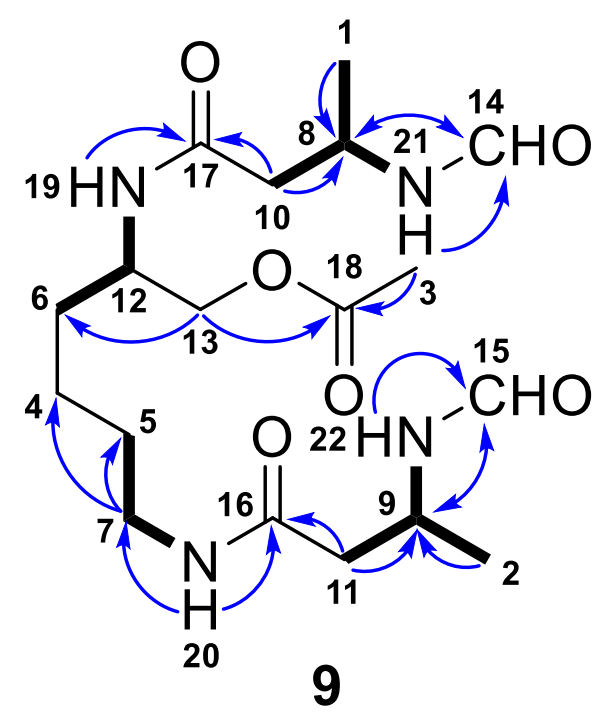


Compound 11 (DMSO- $d_{6}$ )

\begin{tabular}{|l|l|l|l|l|l|}
\hline position & $\delta_{\mathrm{C}}$ & type & $\delta_{\mathrm{H}}(\mathrm{J}$ in $\mathrm{Hz})$ & $\begin{array}{l}{ }^{1} \mathrm{H}-{ }^{1} \mathrm{H} \\
\mathrm{COSY}\end{array}$ & HMBC \\
\hline 1 & 21.48 & $\mathrm{CH}_{3}$ & $1.31, \mathrm{~d}(6.6)$ & $\mathrm{H}-12$ & $\mathrm{C}-9, \mathrm{C}-12$ \\
\hline 2 & 22.19 & $\mathrm{CH}_{2}$ & $1.92(\mathrm{a}), \mathrm{m} ; 1.47(\mathrm{~b}), \mathrm{o}$ & $\mathrm{H}-5, \mathrm{H}-11$ & $\mathrm{C}-5$ \\
\hline 3 & 22.75 & $\mathrm{CH}_{3}$ & $0.85, \mathrm{o}$ & $\mathrm{H}-6$ & $\mathrm{C}-4, \mathrm{C}-6, \mathrm{C}-8$ \\
\hline 4 & 22.77 & $\mathrm{CH}_{3}$ & $0.85, \mathrm{o}$ & $\mathrm{H}-6$ & $\mathrm{C}-3, \mathrm{C}-6, \mathrm{C}-8$ \\
\hline 5 & 23.45 & $\mathrm{CH}_{2}$ & $1.49(\mathrm{a}), \mathrm{o} ; 1.35(\mathrm{~b}), \mathrm{o}$ & $\mathrm{H}-2, \mathrm{H}-13$ & \\
\hline 6 & 27.73 & $\mathrm{CH}$ & $1.48, \mathrm{o}$ & $\mathrm{H}-3, \mathrm{H}-4$ & $\mathrm{C}-3, \mathrm{C}-4, \mathrm{C}-8$ \\
\hline 7 & 33.90 & $\mathrm{CH}_{2}$ & $2.07, \mathrm{~m}$ & $\mathrm{H}-8$ & $\mathrm{C}-6, \mathrm{C}-8, \mathrm{C}-17$ \\
\hline 8 & 34.80 & $\mathrm{CH}_{2}$ & $1.38, \mathrm{o}$ & $\mathrm{H}-6, \mathrm{H}-7$ & $\mathrm{C}-3, \mathrm{C}-4, \mathrm{C}-6, \mathrm{C}-7, \mathrm{C}-17$ \\
\hline 9 & 42.44 & $\mathrm{CH}_{2}$ & $2.61, \mathrm{~m} ; 2.44, \mathrm{~m}$ & $\mathrm{H}-12$ & $\mathrm{C}-1, \mathrm{C}-12, \mathrm{C}-16$ \\
\hline 10 & 43.75 & $\mathrm{CH}$ & $3.08, \mathrm{~m}$ & $\mathrm{H}-13, \mathrm{H}-19$ & $\mathrm{C}-5, \mathrm{C}-13, \mathrm{C}-17$ \\
\hline 11 & 46.89 & $\mathrm{CH}$ & $3.64, \mathrm{~m}$ & $\mathrm{H}-2, \mathrm{H}-20$ & \\
\hline 12 & 47.68 & $\mathrm{CH}$ & $4.07, \mathrm{~m}$ & $\mathrm{H}-1, \mathrm{H}-9$ & $\mathrm{C}-1, \mathrm{C}-9$ \\
\hline 13 & 66.63 & $\mathrm{CH}$ & $3.87, \mathrm{~m}$ & $\mathrm{H}-5, \mathrm{H}-10$ & \\
\hline 14 & 92.26 & $\mathrm{CH}$ & $4.79, \mathrm{~s}$ & $\mathrm{H}-11, \mathrm{H}-18$ & $\mathrm{C}-2, \mathrm{C}-13$ \\
\hline 15 & 155.41 & $\mathrm{C}$ & & & \\
\hline 16 & 168.44 & $\mathrm{C}$ & & & \\
\hline 17 & 172.79 & $\mathrm{C}$ & & $\mathrm{H}-14$ & \\
\hline 18 & & $\mathrm{OH}$ & $6.34, \mathrm{~s}$ & $\mathrm{H}-10$ & $\mathrm{C}-17$ \\
\hline 19 & & $\mathrm{NH}$ & $7.74, \mathrm{o}$ & $\mathrm{H}-11$ & $\mathrm{C}-16$ \\
\hline 20 & & $\mathrm{NH}$ & $8.04, \mathrm{~d}(7.9)$ & & \\
\hline & & & & & \\
\hline
\end{tabular}

*o: overlapped

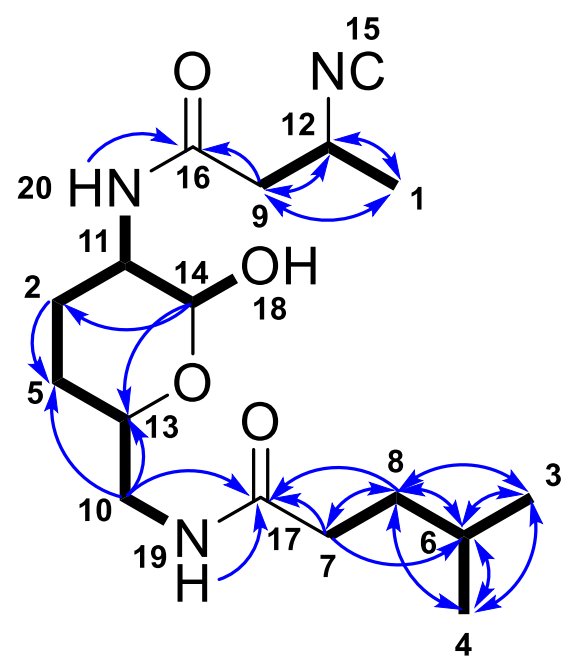


Compound 12 (DMSO- $d_{6}$ )

\begin{tabular}{|c|c|c|c|c|c|c|}
\hline position & $\delta_{\mathrm{C}}$ & type & $\delta_{\mathrm{H}}(J$ in $\mathrm{Hz})$ & $\begin{array}{l}{ }^{1} \mathrm{H}-{ }^{1} \mathrm{H} \\
\mathrm{COSY} \\
\end{array}$ & HMBC & ROESY \\
\hline 1 & 17.92 & $\mathrm{CH}_{3}$ & $1.59, \mathrm{~s}$ & $\mathrm{H}-6, \mathrm{H}-13$ & $\mathrm{C}-5, \mathrm{C}-13, \mathrm{C}-14, \mathrm{C}-17$ & H-6 \\
\hline 2 & 21.06 & $\mathrm{CH}_{3}$ & $1.31, \mathrm{~d}(6.6)$ & $\mathrm{H}-10$ & $\mathrm{C}-7, \mathrm{C}-10$ & $\mathrm{H}-10$ \\
\hline 3 & 21.76 & $\mathrm{CH}_{2}$ & $\begin{array}{l}1.94(\mathrm{a}), \mathrm{m} ; 1.49 \\
(\mathrm{~b}), \mathrm{o}\end{array}$ & H-9 & C-4, C-9, C-11 & H-9, H-11 \\
\hline 4 & 23.06 & $\mathrm{CH}_{2}$ & $\begin{array}{l}1.47 \text { (a), o; } 1.34 \\
\text { (b), o }\end{array}$ & $\mathrm{H}-3$ & & \\
\hline 5 & 25.52 & $\mathrm{CH}_{3}$ & $1.68, \mathrm{~s}$ & $\mathrm{H}-6, \mathrm{H}-13$ & $\mathrm{C}-1, \mathrm{C}-13, \mathrm{C}-14, \mathrm{C}-17$ & $\mathrm{H}-13$ \\
\hline 6 & 35.14 & $\mathrm{CH}_{2}$ & $2.82, \mathrm{~d}(7.7)$ & $\mathrm{H}-5, \mathrm{H}-13$ & C-13, C-14, C-17 & $\begin{array}{l}\mathrm{H}-1, \mathrm{H}-13, \\
\mathrm{H}-18\end{array}$ \\
\hline 7 & 42.00 & $\mathrm{CH}_{2}$ & $\begin{array}{l}\text { 2.61, dd (14.4, } \\
8.7) ; 2.44, \text { o }\end{array}$ & $\mathrm{H}-10$ & $C-2, C-10, C-16$ & $\begin{array}{l}\text { H-2, H-10, } \\
\text { H-19 }\end{array}$ \\
\hline 8 & 43.43 & $\mathrm{CH}_{2}$ & $3.08, \mathrm{~m}$ & $\mathrm{H}-11, \mathrm{H}-18$ & $\mathrm{C}-3, \mathrm{C}-11, \mathrm{C}-17$ & $\mathrm{H}-11$ \\
\hline 9 & 46.45 & $\mathrm{CH}$ & $3.64, \mathrm{~m}$ & H-4. H-19 & $\mathrm{C}-4, \mathrm{C}-12, \mathrm{C}-16$ & $\mathrm{H}-3 \mathrm{a}, \mathrm{H}-12$ \\
\hline 10 & 47.23 & $\mathrm{CH}$ & $4.08, \mathrm{~m}$ & $\mathrm{H}-2, \mathrm{H}-7$ & C-2, C-7, C-15, C-16 & $\mathrm{H}-2, \mathrm{H}-7$ \\
\hline 11 & 66.21 & $\mathrm{CH}$ & $3.87, \mathrm{~m}$ & $\mathrm{H}-3, \mathrm{H}-8$ & $C-3, C-8, C-12$ & $\begin{array}{l}\text { H-3a, H-4b, } \\
\text { H-8, H-12 }\end{array}$ \\
\hline 12 & 91.82 & $\mathrm{CH}$ & $4.79, \mathrm{~s}$ & $\mathrm{H}-9$ & $\mathrm{C}-3, \mathrm{C}-11$ & $\mathrm{H}-9$ \\
\hline 13 & 118.53 & $\mathrm{CH}$ & $5.23, \mathrm{~m}$ & $\mathrm{H}-5, \mathrm{H}-6$ & C-1, C-5, C-6, C-17 & $\mathrm{H}-5, \mathrm{H}-6$ \\
\hline 14 & 133.04 & $\mathrm{C}$ & & & & \\
\hline 15 & 154.86 & $\mathrm{C}$ & & & & \\
\hline 16 & 168.06 & $\mathrm{C}$ & & & & \\
\hline 17 & 170.79 & $\mathrm{C}$ & & & & \\
\hline 18 & & $\mathrm{NH}$ & $7.75, \mathrm{t}(5.8)$ & $\mathrm{H}-8$ & C-8, C-17 & $\begin{array}{l}\mathrm{H}-6, \mathrm{H}-8, \mathrm{H}- \\
11\end{array}$ \\
\hline 19 & & $\mathrm{NH}$ & $8.07, \mathrm{~d}(7.9)$ & H-9 & C-9, C-12, C-16 & $\begin{array}{l}\text { H-4, H-7, H- } \\
9, \mathrm{H}-12\end{array}$ \\
\hline
\end{tabular}

${ }^{*}$ o: overlapped

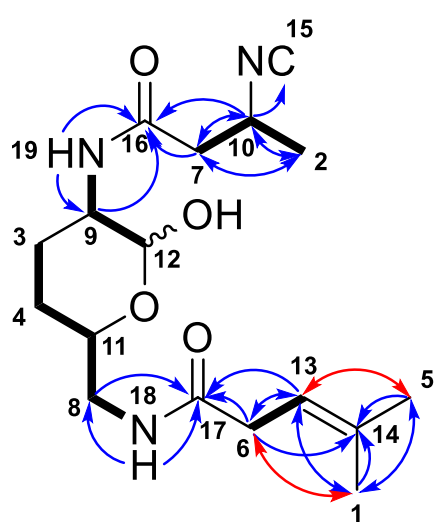

12 
Table S2. ${ }^{1} \mathrm{H}(600 \mathrm{MHz})$ and ${ }^{13} \mathrm{C}(150 \mathrm{MHz})$ NMR Data of the pyran ring of SF2768 and its analogues.

\begin{tabular}{|c|c|c|c|c|c|c|}
\hline \multicolumn{7}{|c|}{$\mathrm{SF} 2768^{1}\left(\mathrm{D}_{2} \mathrm{O}\right)$} \\
\hline & \multicolumn{2}{|r|}{$\beta$-anomer } & \multicolumn{2}{|r|}{$\alpha$-anomer } & \multirow[b]{2}{*}{$\Delta \delta_{\mathrm{C}}$} & \\
\hline Position & $\delta_{\mathrm{C}}$ & $\delta_{\mathrm{H}}$ & $\delta_{\mathrm{C}}$ & $\delta_{\mathrm{H}}$ & & $\Delta \delta_{\mathrm{H}}$ \\
\hline 1 & 95.0 & 4.93 & 92.9 & 5.06 & 2.1 & -0.13 \\
\hline 2 & 48.8 & 4.04 & 48.0 & 3.84 & 0.8 & 0.20 \\
\hline 3 & 27.3 & $1.92(\mathrm{a}), 1.84(\mathrm{~b})$ & 22.3 & $2.06(a), 1.74(b)$ & 5.0 & - \\
\hline 4 & 22.9 & $1.51(\mathrm{a}), 1.47(\mathrm{~b})$ & 23.3 & 1.59 & -0.4 & - \\
\hline 5 & 75.9 & 3.77 & 68.3 & 4.11 & 7.6 & -0.34 \\
\hline 6 & 44.2 & $3.42(\mathrm{a}), 3.34(\mathrm{~b})$ & 44.2 & 3.34 & 0.0 & - \\
\hline \multicolumn{7}{|c|}{ SF2768 $\left(\mathrm{DMSO}-d_{6}\right)$} \\
\hline & \multicolumn{2}{|r|}{$\beta$-anomer } & \multicolumn{2}{|r|}{$\alpha$-anomer } & & \\
\hline & $\delta_{\mathrm{C}}$ & $\delta_{\mathrm{H}}$ & $\delta_{\mathrm{C}}$ & $\delta_{\mathrm{H}}$ & $\Delta \delta_{\mathrm{C}}$ & $\Delta \delta_{\mathrm{H}}$ \\
\hline 1 & 94.2 & 4.69 & 91.9 & 4.79 & 2.3 & -0.10 \\
\hline 2 & 47.4 & 3.83 & 46.4 & 3.64 & 1.0 & 0.19 \\
\hline 3 & 26.5 & $1.72(\mathrm{a}), 1.61(\mathrm{~b})$ & 21.5 & $1.92(\mathrm{a}), 1.46(\mathrm{~b})$ & 5.0 & - \\
\hline 4 & 22.4 & $1.37(\mathrm{a}), 1.30(\mathrm{~b})$ & 23.0 & $1.49(\mathrm{a}), 1.38(\mathrm{~b})$ & -0.6 & - \\
\hline 5 & 73.7 & 3.52 & 66.1 & 3.88 & 7.6 & -0.36 \\
\hline 6 & 43.3 & $3.30(a), 3.16(b)$ & 43.3 & 3.14 & 0.0 & - \\
\hline \multicolumn{7}{|c|}{ SF2768 B ${ }^{3}\left(\mathrm{DMSO}-d_{6}\right)$} \\
\hline & \multicolumn{2}{|r|}{$\beta$-anomer } & \multicolumn{2}{|r|}{$\alpha$-anomer } & & \\
\hline & $\delta_{\mathrm{C}}$ & $\delta_{\mathrm{H}}$ & $\delta_{\mathrm{C}}$ & $\delta_{\mathrm{H}}$ & $\Delta \delta_{\mathrm{C}}$ & $\Delta \delta_{\mathrm{H}}$ \\
\hline 1 & 94.8 & 4.68 & 92.4 & 4.78 & 2.4 & -0.10 \\
\hline 2 & 47.5 & 3.80 & 46.6 & 3.62 & 0.9 & 0.18 \\
\hline 3 & 26.8 & $1.73(\mathrm{a}), 1.61(\mathrm{~b})$ & 22.1 & $1.92(\mathrm{a}), 1.44(\mathrm{~b})$ & 4.7 & - \\
\hline 4 & 23.0 & 1.33 & 23.4 & $1.51(\mathrm{a}), 1.37(\mathrm{~b})$ & -0.4 & - \\
\hline 5 & 74.2 & 3.51 & 66.5 & 3.88 & 7.7 & -0.37 \\
\hline 6 & 43.6 & $3.28(a), 3.17(b)$ & 43.8 & 3.13 & -0.2 & - \\
\hline \multicolumn{7}{|c|}{ SF2768 C ${ }^{3}\left(\mathrm{DMSO}-d_{6}\right)$} \\
\hline & \multicolumn{2}{|r|}{$\beta$-anomer } & \multicolumn{2}{|r|}{$\alpha$-anomer } & & \\
\hline & $\delta_{\mathrm{C}}$ & $\delta_{\mathrm{H}}$ & $\delta_{\mathrm{C}}$ & $\delta_{\mathrm{H}}$ & $\Delta \delta_{\mathrm{C}}$ & $\Delta \delta_{\mathrm{H}}$ \\
\hline 1 & 94.8 & 4.66 & 92.4 & 4.78 & 2.4 & -0.12 \\
\hline 2 & 47.5 & 3.80 & 46.6 & 3.61 & 0.9 & 0.19 \\
\hline 3 & 26.9 & $1.67(\mathrm{a}), 1.59(\mathrm{~b})$ & 22.1 & $1.90(\mathrm{a}), 1.44(\mathrm{~b})$ & 4.8 & - \\
\hline 4 & 23.1 & 1.34 & 23.4 & $1.49(\mathrm{a}), 1.33(\mathrm{~b})$ & -0.3 & - \\
\hline 5 & 74.3 & 3.40 & 66.6 & 3.87 & 7.7 & -0.47 \\
\hline 6 & 43.5 & $3.23(\mathrm{a}), 3.10(\mathrm{~b})$ & 43.7 & $3.12(a), 3.08(b)$ & -0.2 & - \\
\hline \multicolumn{7}{|c|}{ SF2768 J $\left(\right.$ DMSO- $\left.d_{6}\right)$} \\
\hline & \multicolumn{2}{|r|}{$\beta$-anomer } & \multicolumn{2}{|r|}{$\alpha$-anomer } & & \\
\hline & $\delta_{\mathrm{C}}$ & $\delta_{\mathrm{H}}$ & $\delta_{\mathrm{C}}$ & $\delta_{\mathrm{H}}$ & $\Delta \delta_{\mathrm{C}}$ & $\Delta \delta_{\mathrm{H}}$ \\
\hline 1 & 94.7 & 4.68 & 92.5 & 4.77 & 2.2 & -0.09 \\
\hline
\end{tabular}




\begin{tabular}{|c|c|c|c|c|c|c|}
\hline 2 & 47.6 & 3.78 & 46.6 & 3.61 & 1.0 & 0.07 \\
\hline 3 & 26.2 & $1.70(\mathrm{a}), 1.56(\mathrm{~b})$ & 22.2 & 1.92 & 4.0 & - \\
\hline 4 & 22.9 & 1.33 & 23.5 & $1.46(\mathrm{a}), 1.31(\mathrm{~b})$ & -0.6 & - \\
\hline 5 & 74.1 & 3.51 & 66.6 & 3.86 & 7.5 & -0.34 \\
\hline 6 & 43.3 & $3.29(\mathrm{a}), 3.09(\mathrm{~b})$ & 43.7 & 3.09 & -0.4 & - \\
\hline \multicolumn{7}{|c|}{ SF2768 K (DMSO- $\left.d_{6}\right)$} \\
\hline & \multicolumn{2}{|r|}{$\beta$-anomer } & \multicolumn{2}{|r|}{$\alpha$-anomer } & & \\
\hline & $\delta_{\mathrm{C}}$ & $\delta_{\mathrm{H}}$ & $\delta_{\mathrm{C}}$ & $\delta_{\mathrm{H}}$ & $\Delta \delta_{\mathrm{C}}$ & $\Delta \delta_{\mathrm{H}}$ \\
\hline 1 & 94.7 & 4.68 & 92.3 & 4.79 & 2.4 & -0.11 \\
\hline 2 & 47.6 & 3.81 & 46.9 & 3.64 & 0.7 & 0.17 \\
\hline 3 & 27.0 & $1.72(\mathrm{a}), 1.60(\mathrm{~b})$ & 22.2 & $1.92(\mathrm{a}), 1.47(\mathrm{~b})$ & 4.8 & - \\
\hline 4 & 22.9 & 1.36 & 23.5 & $1.49(\mathrm{a}), 1.35(\mathrm{~b})$ & -0.6 & - \\
\hline 5 & 74.2 & 3.51 & 66.6 & 3.87 & 7.6 & -0.36 \\
\hline 6 & 43.3 & $3.26(\mathrm{a}), 3.10(\mathrm{~b})$ & 43.7 & 3.09 & -0.4 & - \\
\hline \multicolumn{7}{|c|}{ SF2768 L (DMSO- $\left.d_{6}\right)$} \\
\hline & \multicolumn{2}{|r|}{$\beta$-anomer } & \multicolumn{2}{|r|}{$\alpha$-anomer } & & \\
\hline & $\delta_{\mathrm{C}}$ & $\delta_{\mathrm{H}}$ & $\delta_{\mathrm{C}}$ & $\delta_{\mathrm{H}}$ & $\Delta \delta_{\mathrm{C}}$ & $\Delta \delta_{\mathrm{H}}$ \\
\hline 1 & 94.2 & 4.67 & 91.8 & 4.79 & 2.4 & -0.12 \\
\hline 2 & 47.4 & 3.82 & 46.5 & 3.64 & 0.9 & 0.18 \\
\hline 3 & 26.5 & $1.70(\mathrm{a}), 1.60(\mathrm{~b})$ & 21.8 & $1.94(\mathrm{a}), 1.49(\mathrm{~b})$ & 4.7 & - \\
\hline 4 & 22.5 & 1.35 & 23.1 & $1.47(\mathrm{a}), 1.34(\mathrm{~b})$ & -0.6 & - \\
\hline 5 & 73.9 & 3.50 & 66.2 & 3.87 & 7.7 & -0.37 \\
\hline 6 & 43.3 & $3.25(\mathrm{a}), 3.09(\mathrm{~b})$ & 43.4 & 3.08 & -0.1 & - \\
\hline
\end{tabular}
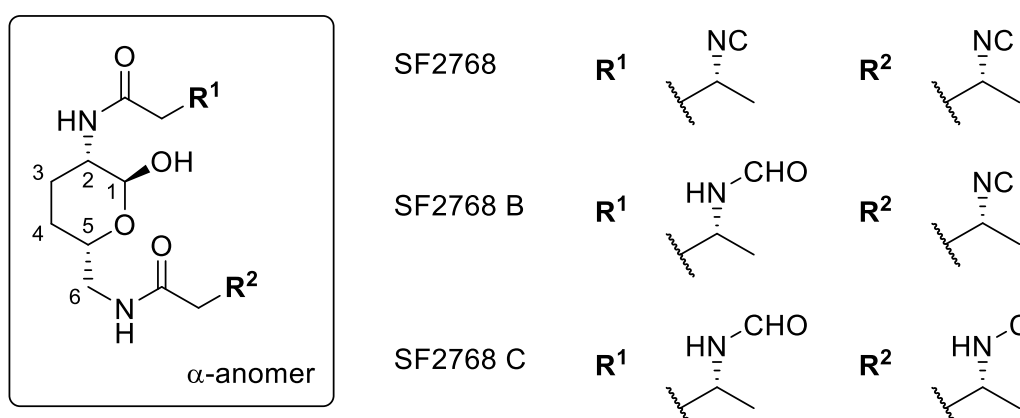

$\mathrm{SF} 2768 \mathrm{~B}$

$\mathrm{SF} 2768 \mathrm{C}$

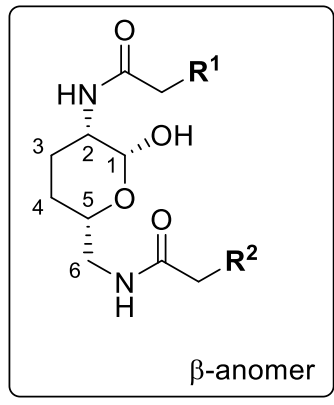

SF2768 J<smiles>[R1]C(C)C(C)C</smiles><smiles>[R]CC(C)C(C)C</smiles>

SF2768 K<smiles>[R]CC(C)C(C)N</smiles><smiles>[R]C(C)CC(C)C(C)C</smiles>

SF2768 L<smiles>[R]CC(C)C(C)N</smiles><smiles>[R]C1CC=C(C)C1</smiles> 
Table S3. The detailed data of the ICP-MS analysis.

\begin{tabular}{cccc}
\hline Sample & $\begin{array}{c}\text { Dry cell weight } \\
(\mathrm{g})\end{array}$ & $\begin{array}{c}\text { Concentration of }{ }^{63} \mathrm{Cu} \\
(\mu \mathrm{g} / \mathrm{L})\end{array}$ & $\begin{array}{c}\text { Final volume of lysate } \\
(\mathrm{mL})\end{array}$ \\
\hline SF2768-treated-1 & 0.0106 & 4.445 & 10 \\
SF2768-treated-2 & 0.0106 & 4.419 & 10 \\
SF2768-treated-3 & 0.0110 & 4.649 & 10 \\
SF2768-treated-4 & 0.0110 & 4.075 & 10 \\
SF2768-treated-5 & 0.0115 & 3.792 & 10 \\
CK-1 & 0.0108 & 5.419 & 10 \\
CK-2 & 0.0108 & 5.450 & 10 \\
CK-3 & 0.0108 & 5.851 & 10 \\
CK-4 & 0.0105 & 6.024 & 10 \\
CK-5 & 0.0107 & 5.394 & 10 \\
\hline
\end{tabular}


A

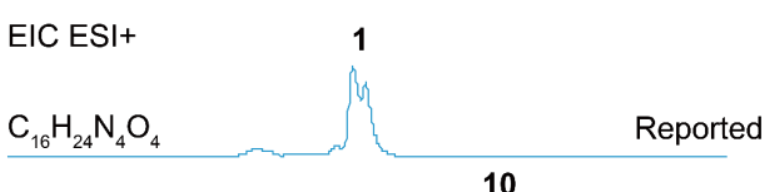

$\mathrm{C}_{16} \mathrm{H}_{30} \mathrm{~N}_{2} \mathrm{O}_{4}$

Reported

12

12

$\mathrm{m} / \mathrm{z} 338.21$

$\mathrm{C}_{17} \mathrm{H}_{27} \mathrm{~N}_{3} \mathrm{O}_{4}$

This study

11

$\mathrm{m} / \mathrm{z} 340.22$

$\mathrm{C}_{17} \mathrm{H}_{29} \mathrm{~N}_{3} \mathrm{O}_{4}$

This study

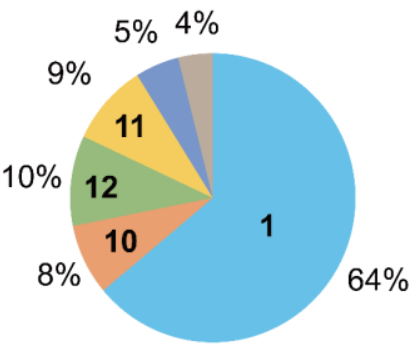

$\mathrm{m} / \mathrm{z} 340.22$

$\mathrm{C}_{17} \mathrm{H}_{29} \mathrm{~N}_{3} \mathrm{O}_{4}$

$?$

Unkown

$\mathrm{m} / \mathrm{z} 326.21$

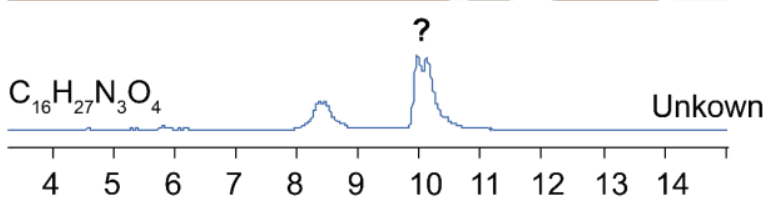

B

Acquisition Time (min)
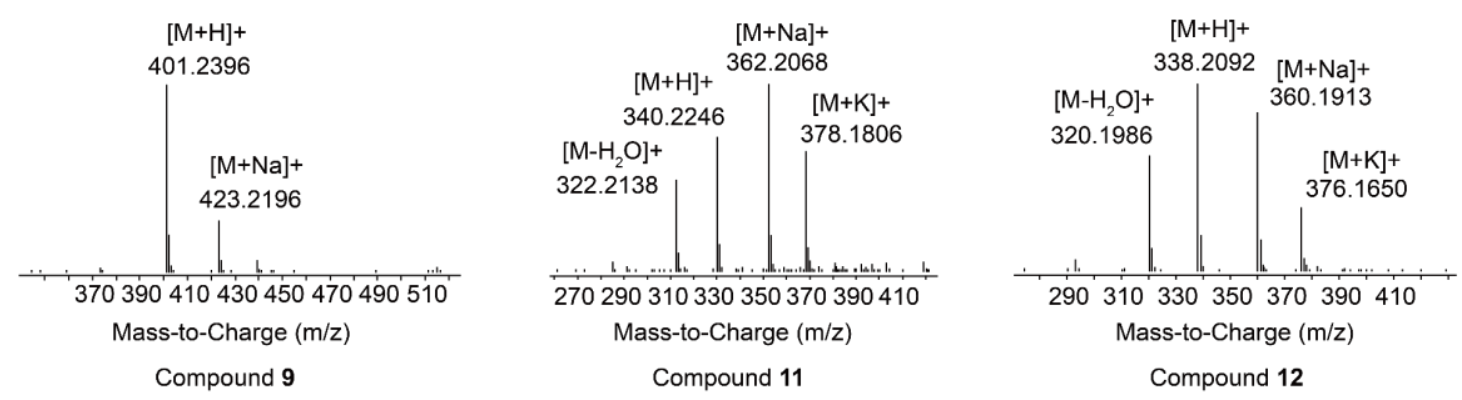

Figure S1. (A) Detection of the SF2768 analogues in the fermentation broth of $S$. lividans::p13C. (B) HR-ESI-MS analysis of compound 9, 11 and 12. 


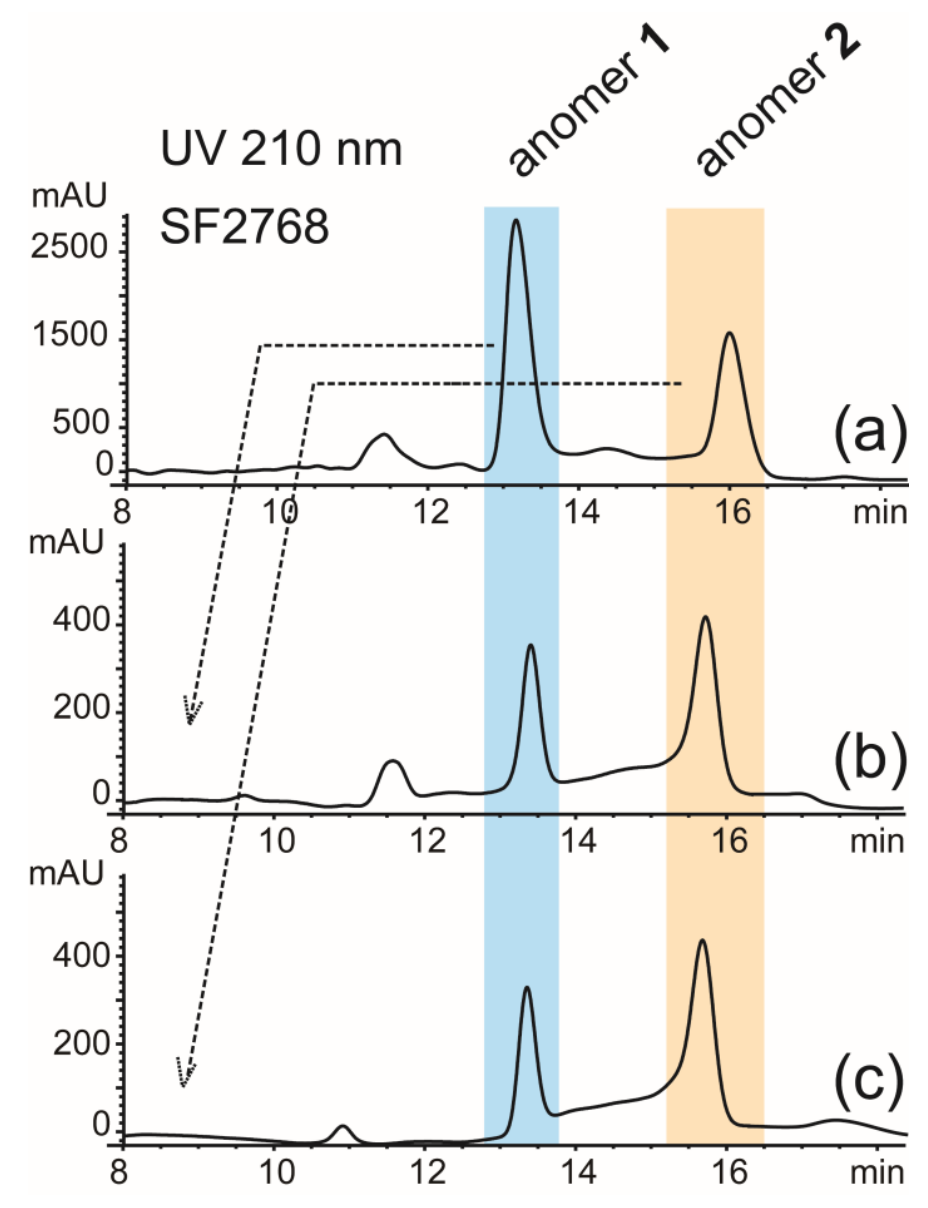

Figure S2. Detection of the anomers of SF2768 on HPLC. Collection and reinjection of the two distinct peaks of SF2768 (1) on HPLC. (a) SF2768 standard. (b) reinjection of the anomer 1 (blue). (c) reinjection of the anomer 2 (orange). A spontaneous interconversion of the anomers was observed in the acetonitrile-water mobile phase (20\%, isocratic, $1 \mathrm{~mL} / \mathrm{min})$. Agilent 1260 system, C18 column $(4.6 \times 250 \mathrm{~mm}, 5 \mu \mathrm{m}$ particle size, Kromasil). 


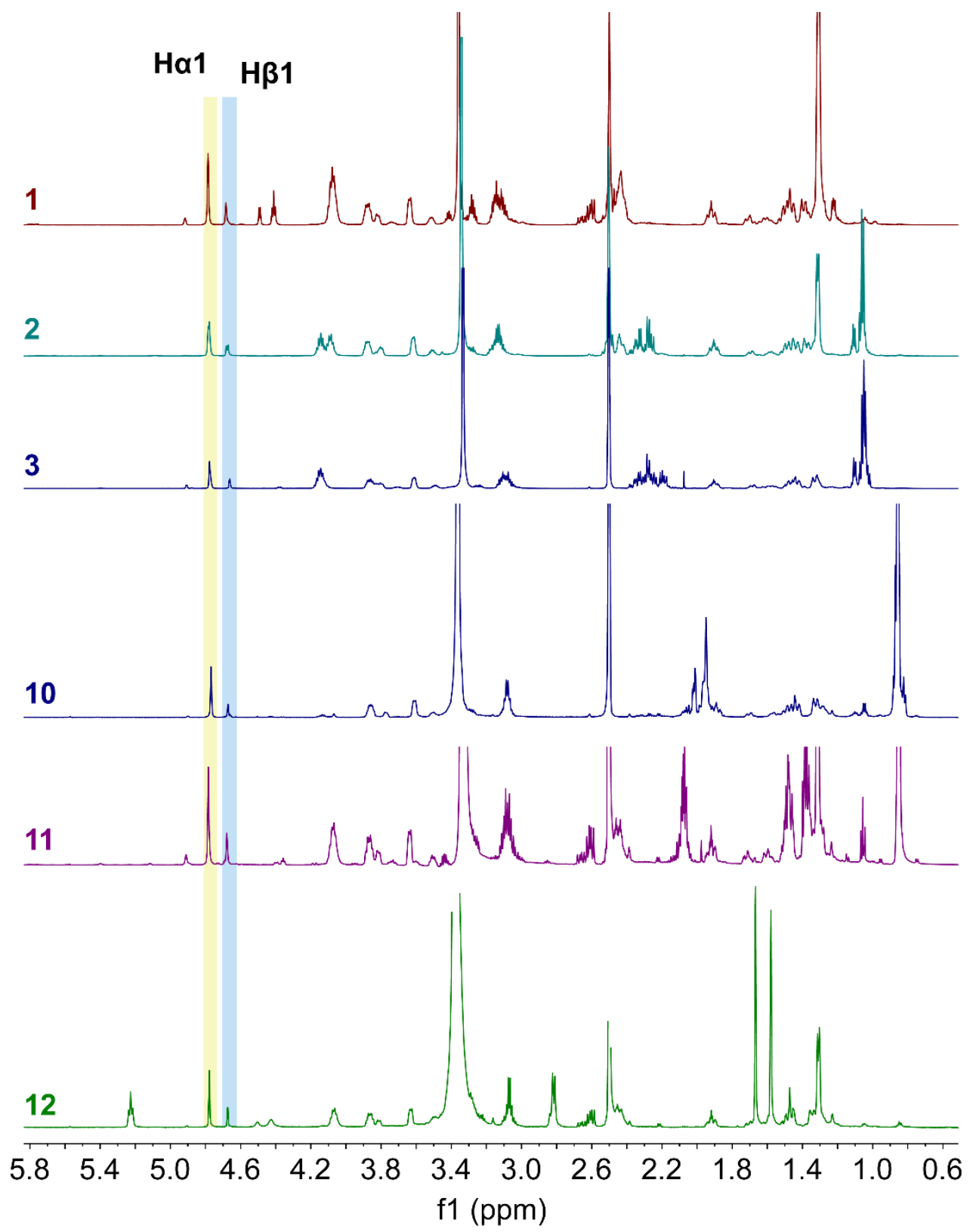

Figure S3. The anomeric protons of SF2768 (1) and its analogues. 
P. aeruginosa ATCC 27853

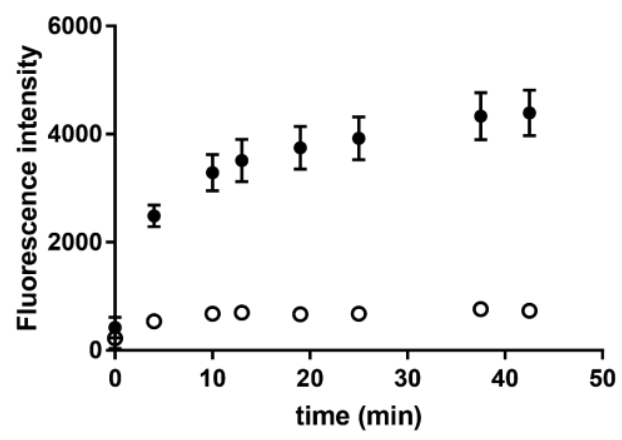

B. subtilis 168

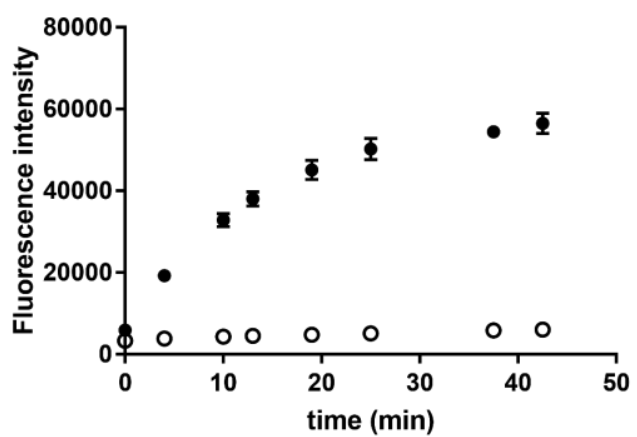

M. smegmatis $\mathrm{mc}^{2} 155$

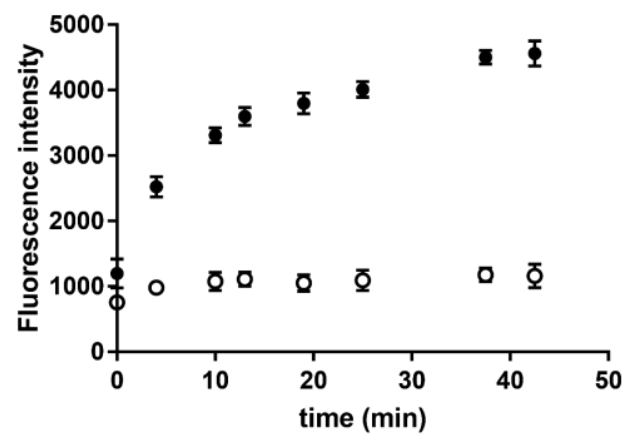

E. coli BL21

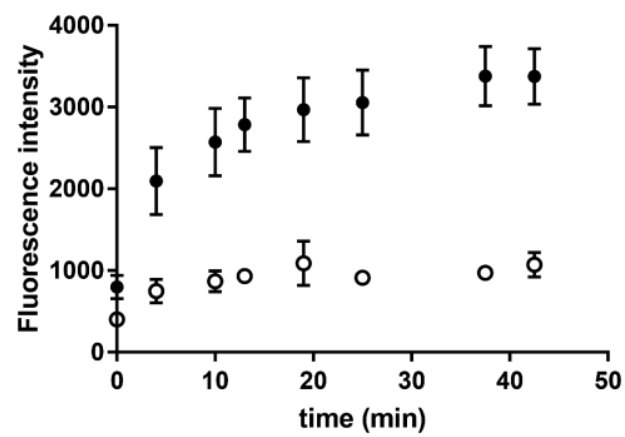

E. faecalis ATCC 29212

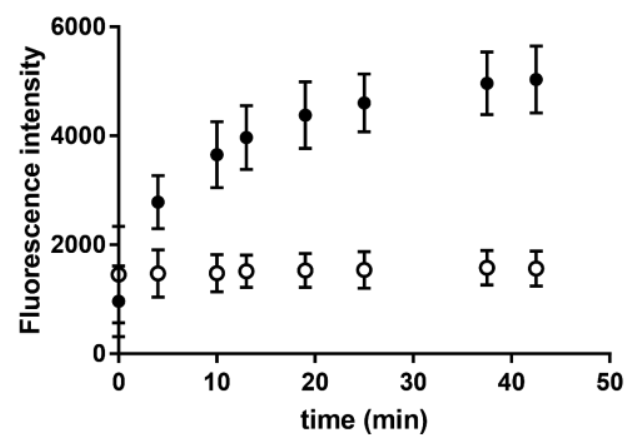

A. baumannii ATCC 19606

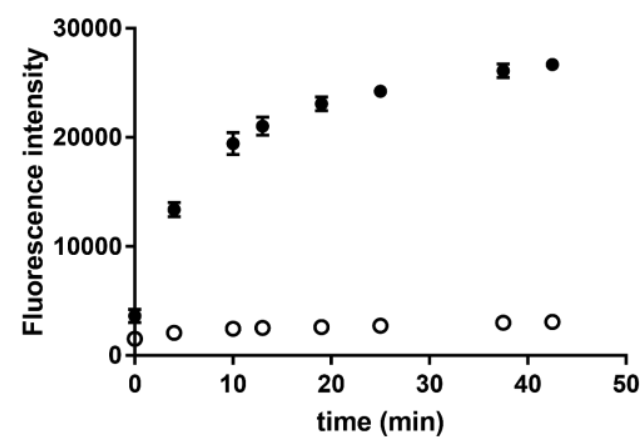

S. aureus ATCC 43300

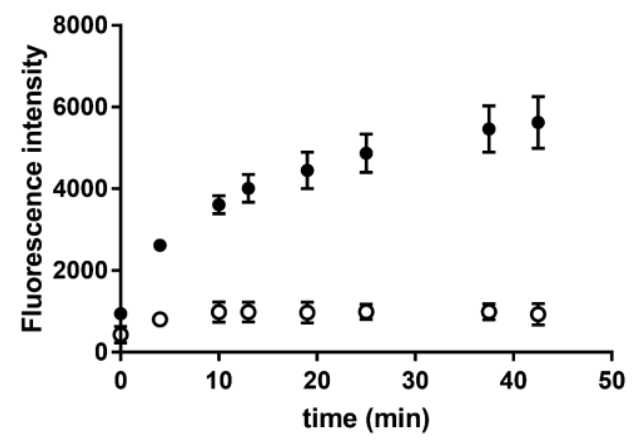

K. pneumoniae CMCC 46117

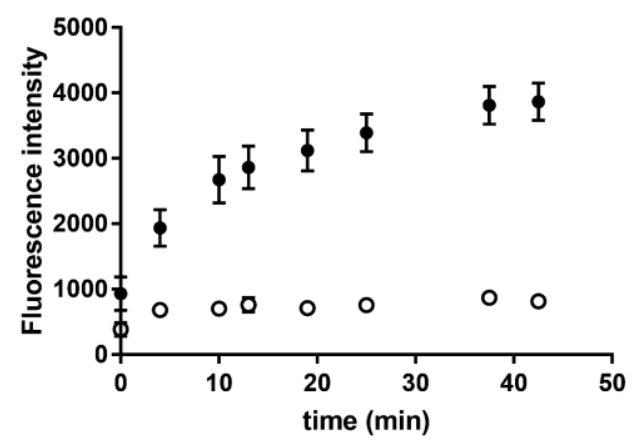

Figure S4. Time-course of the ROS accumulation in eight bacteria. 
Figure S5. NMR spectra of 9 in DMSO- $d_{6}$ at $600 \mathrm{MHz}$ and $151 \mathrm{MHz}$.

A. ${ }^{1} \mathrm{H}$ spectrum

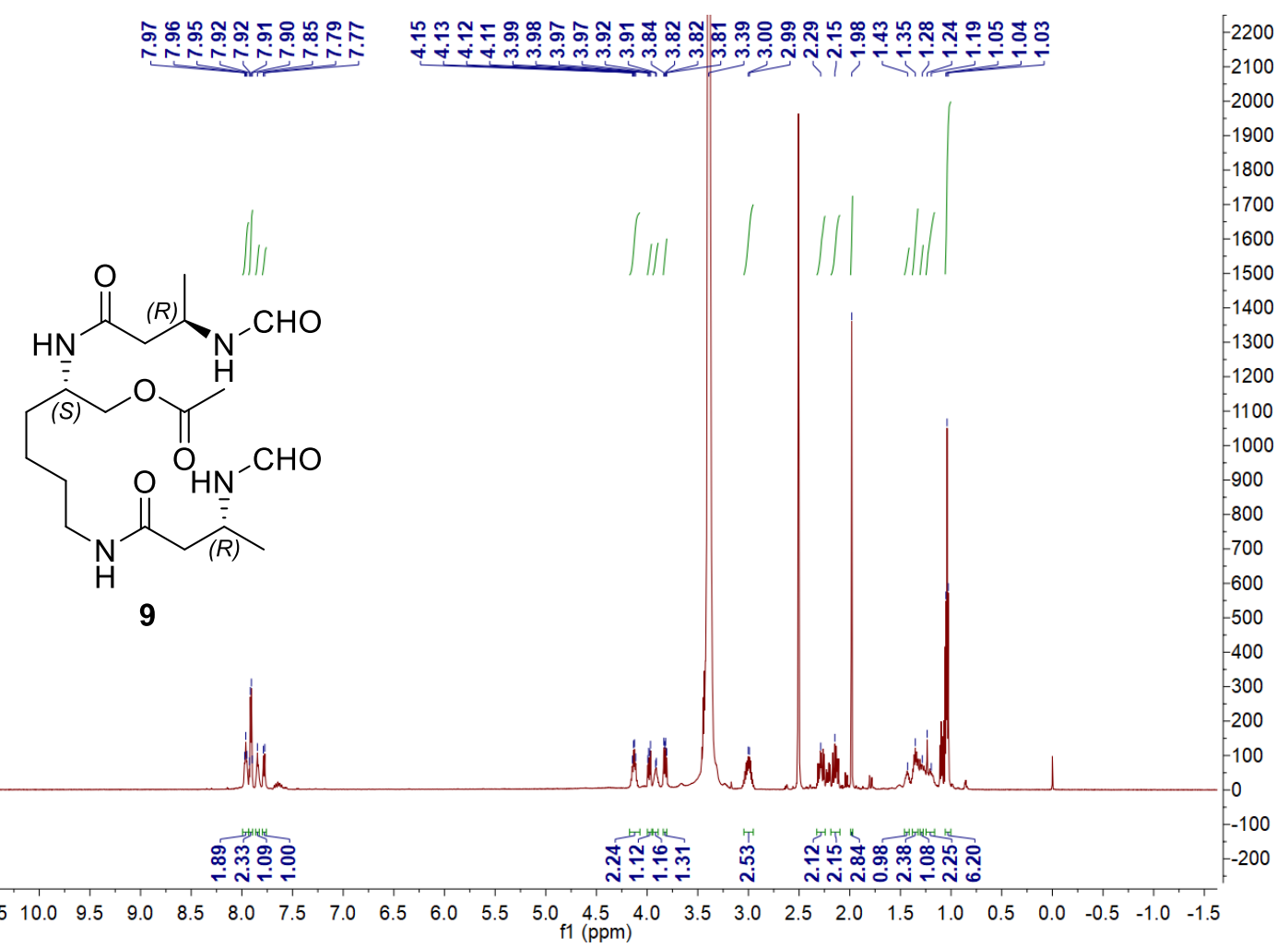

B. ${ }^{13} \mathrm{C}$ spectrum

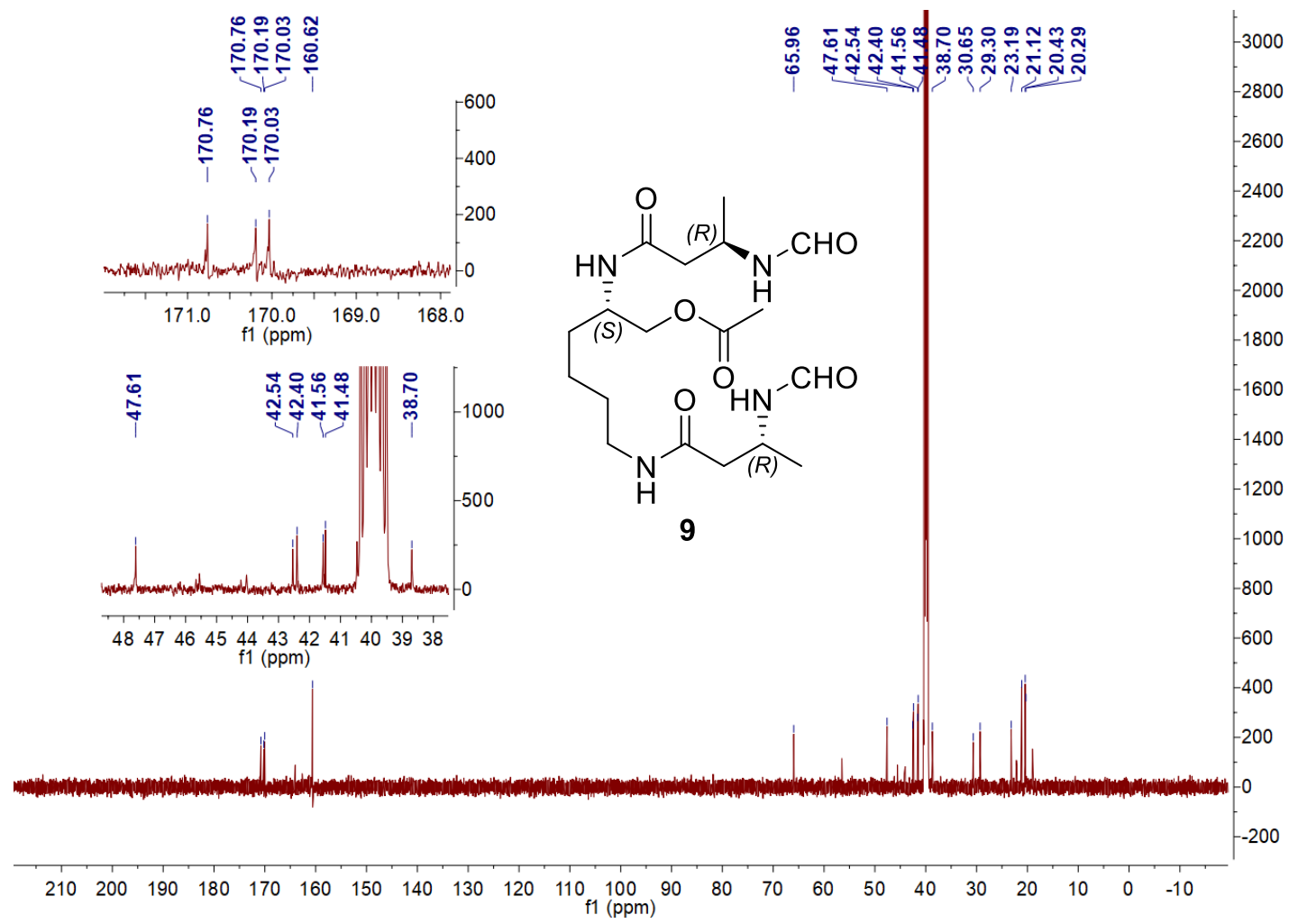


C. DEPT 135 spectrum
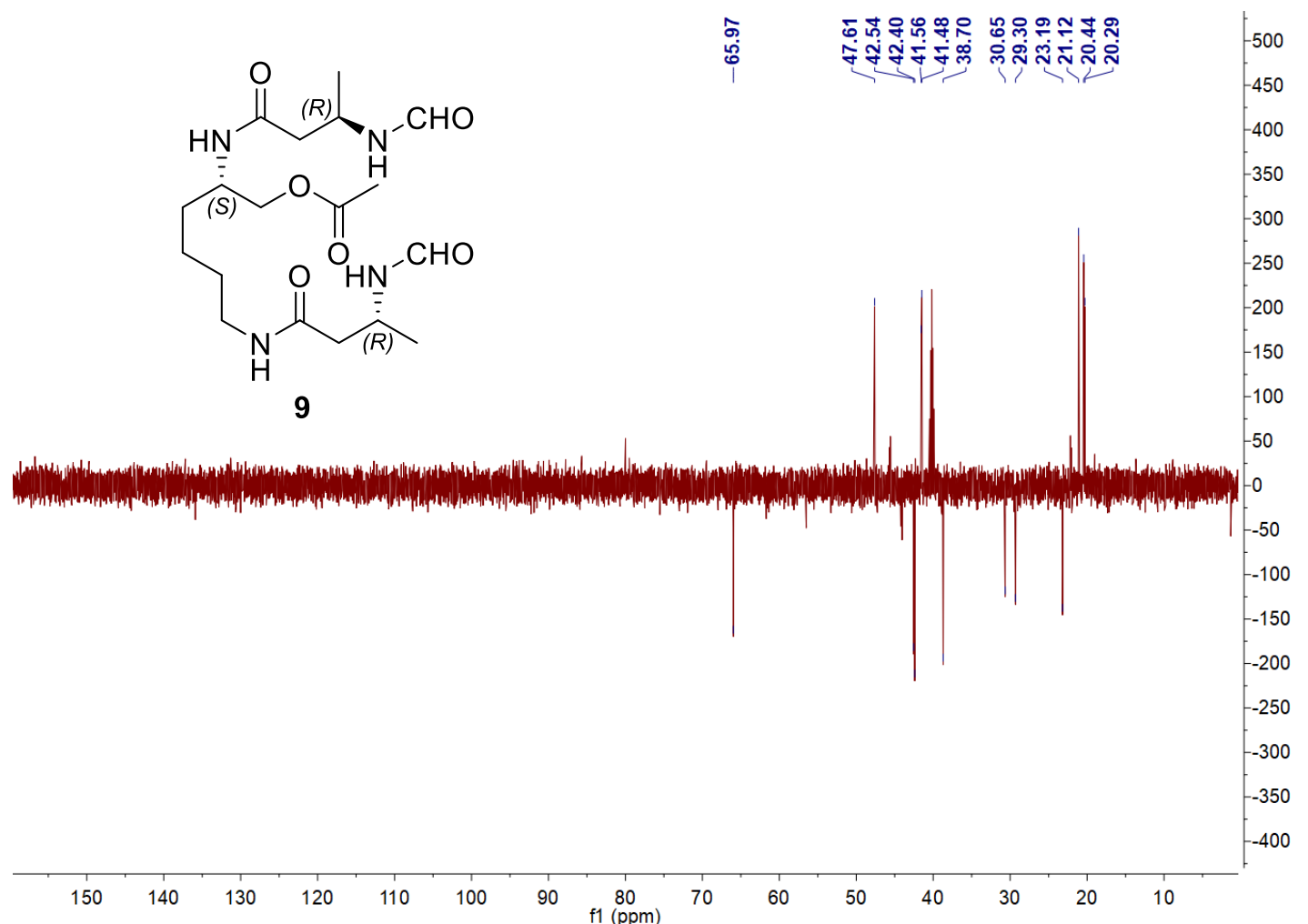

\section{COSY spectrum}

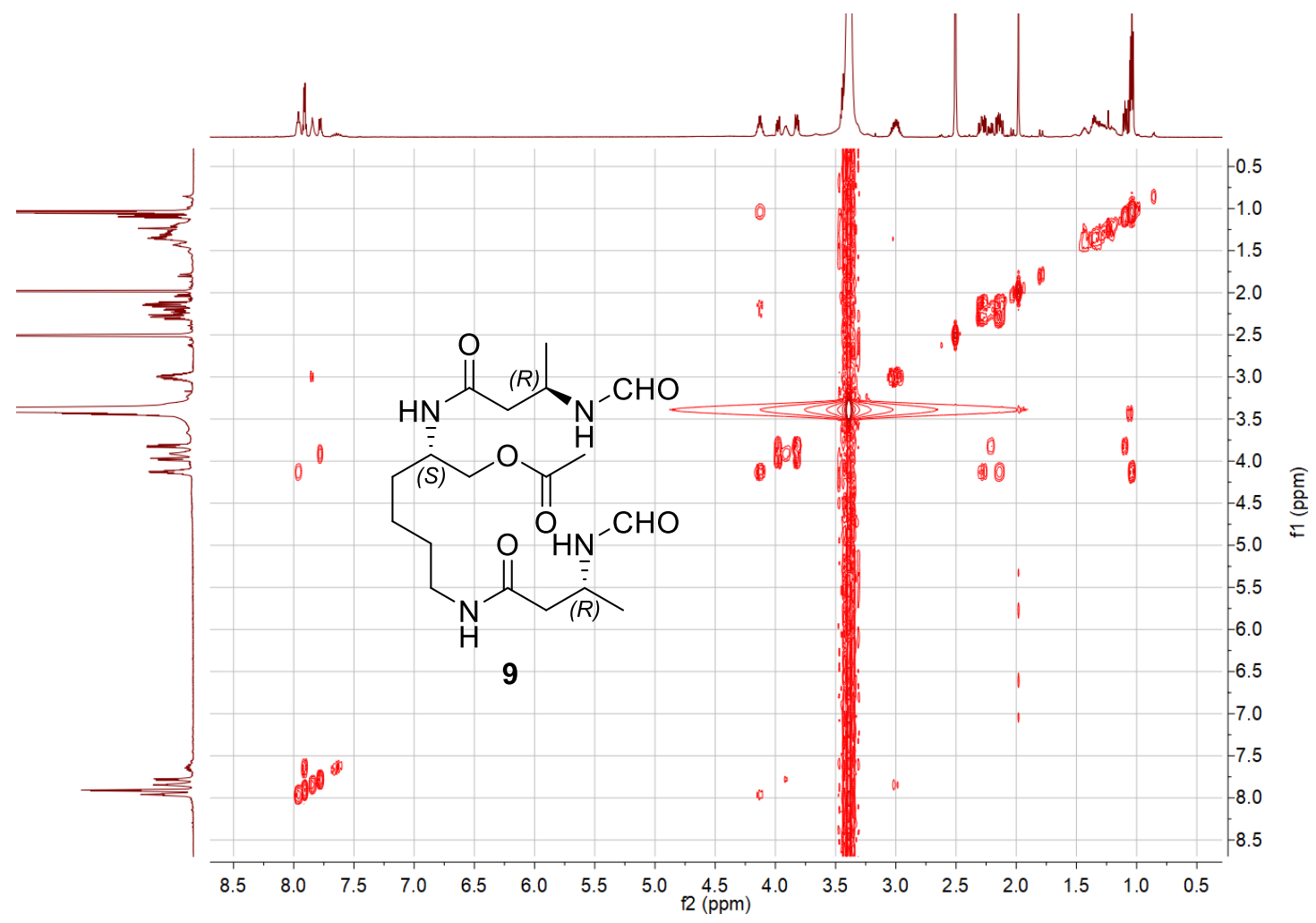


E. HSQC spectrum

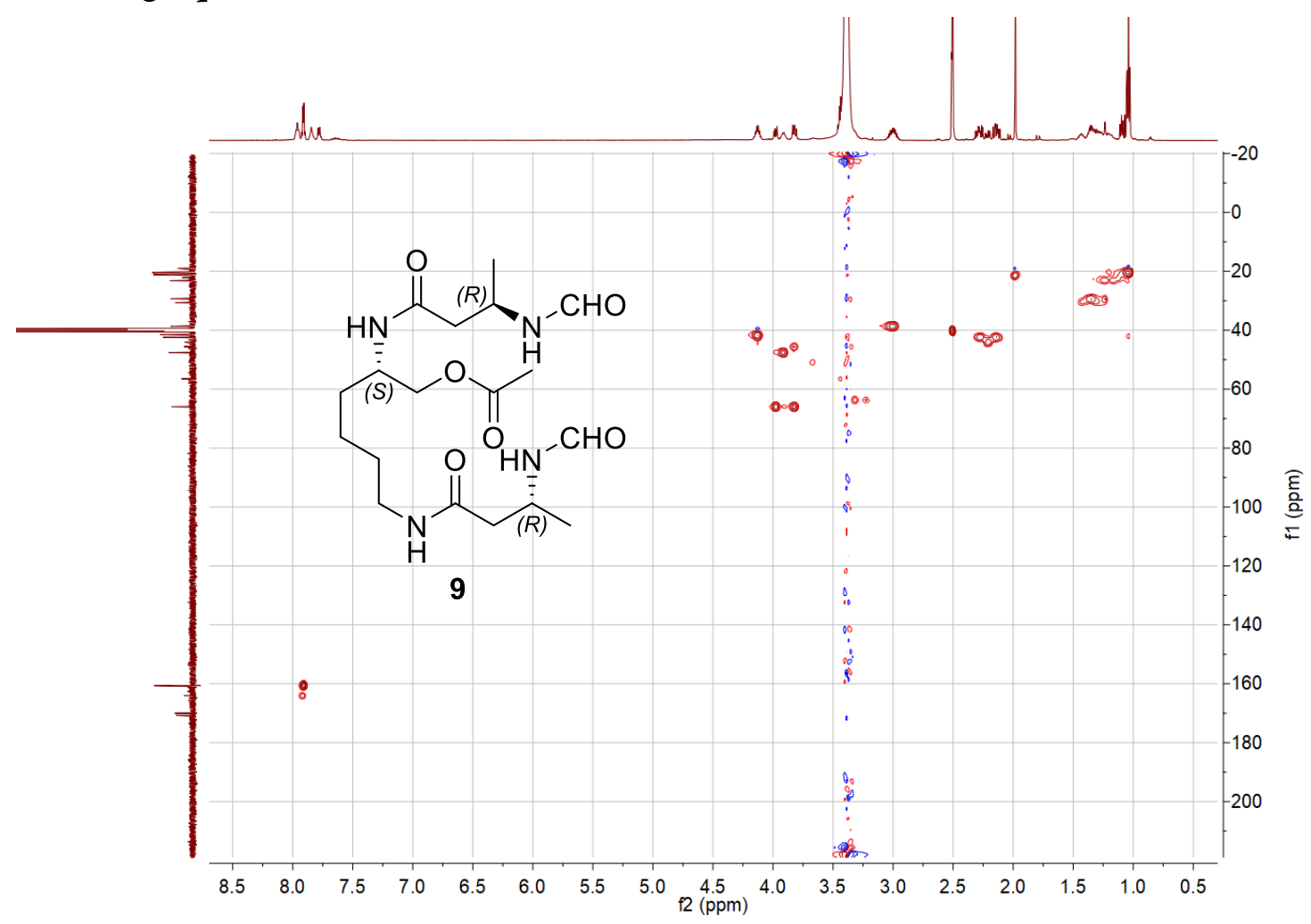

F. HMBC spectrum

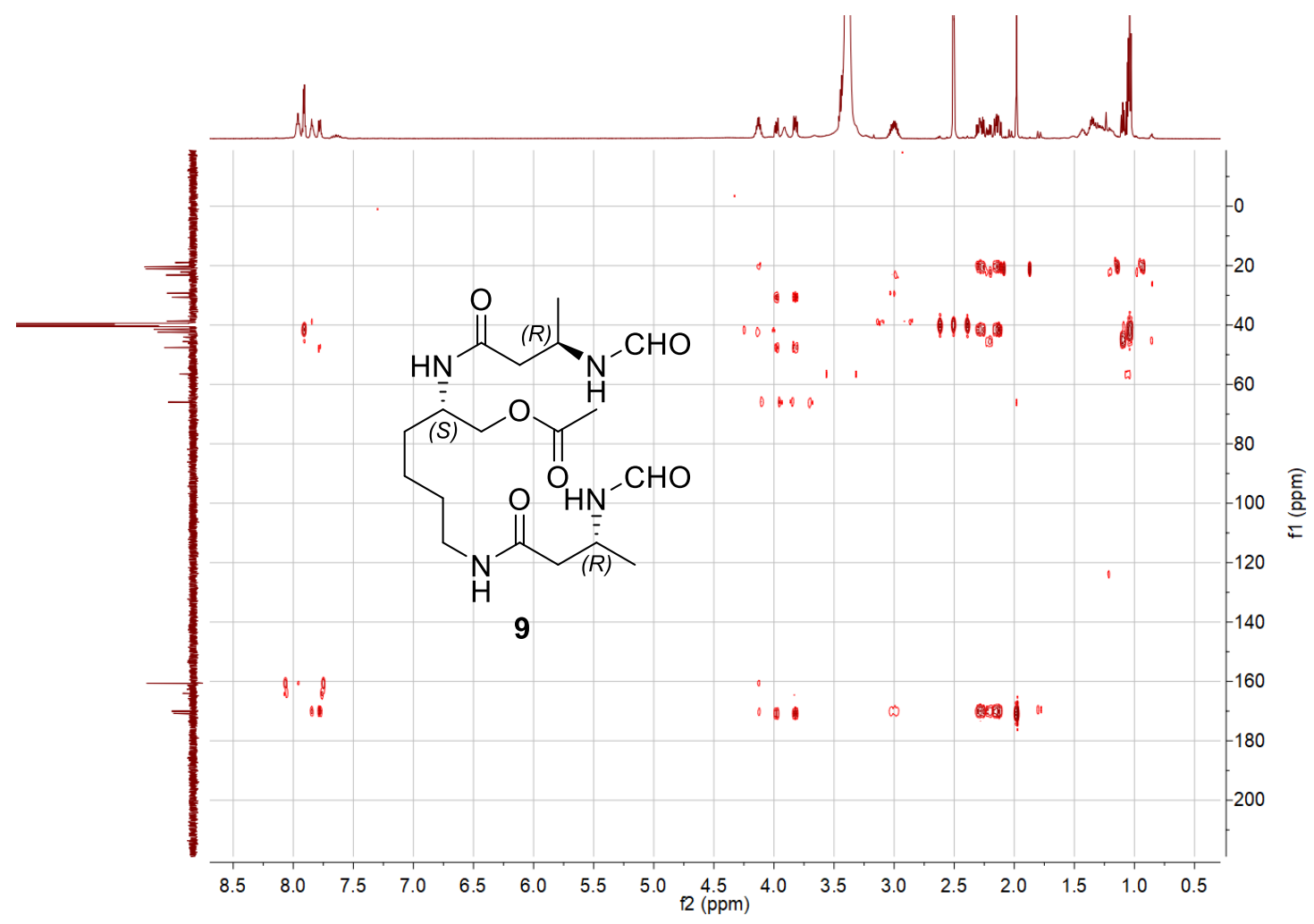


Figure S6. NMR spectra of 11 in DMSO- $d_{6}$ at $600 \mathrm{MHz}$ and $151 \mathrm{MHz}$.

A. ${ }^{1} \mathrm{H}$ spectrum

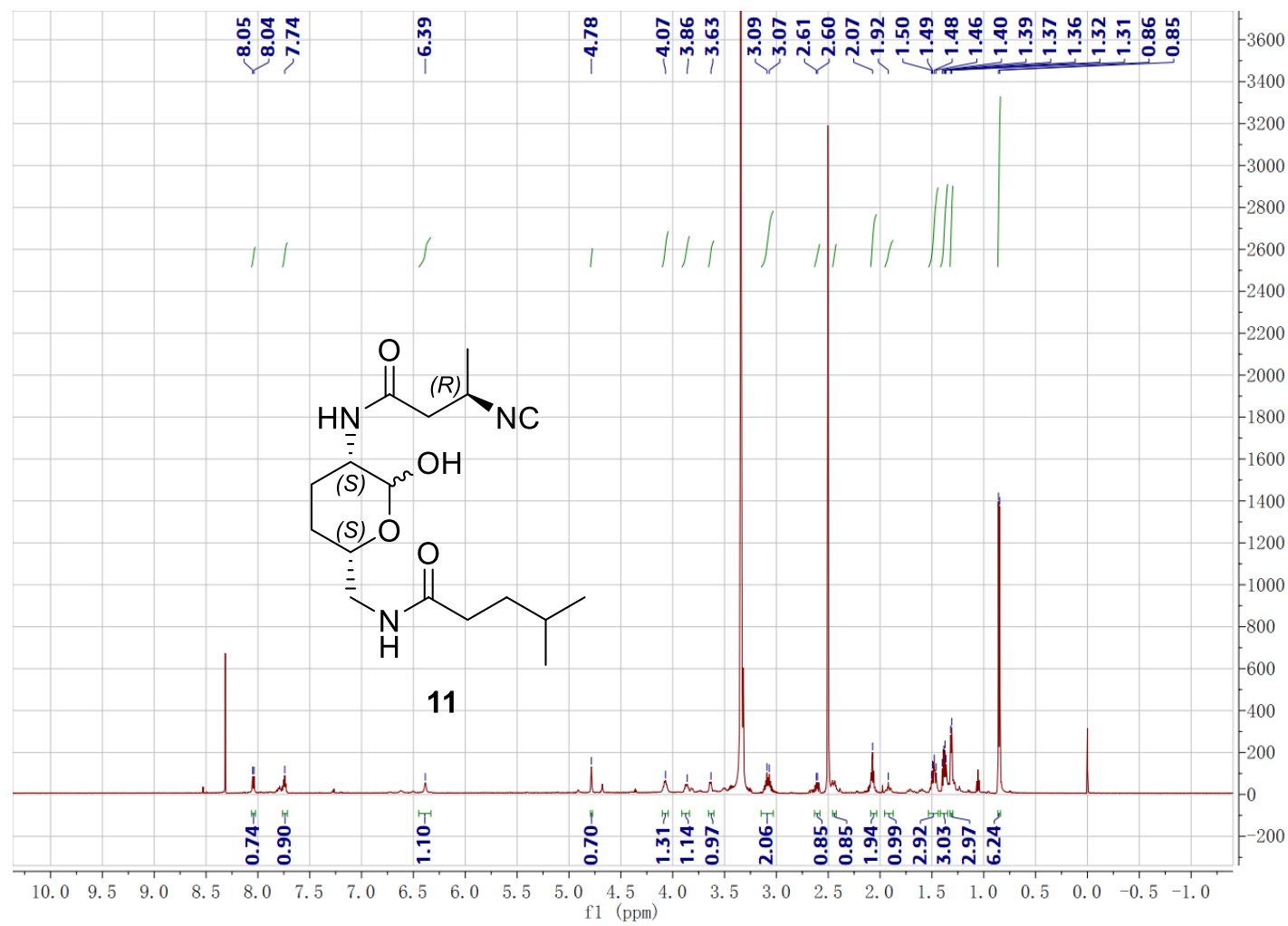

B. ${ }^{13} \mathrm{C}$ spectrum

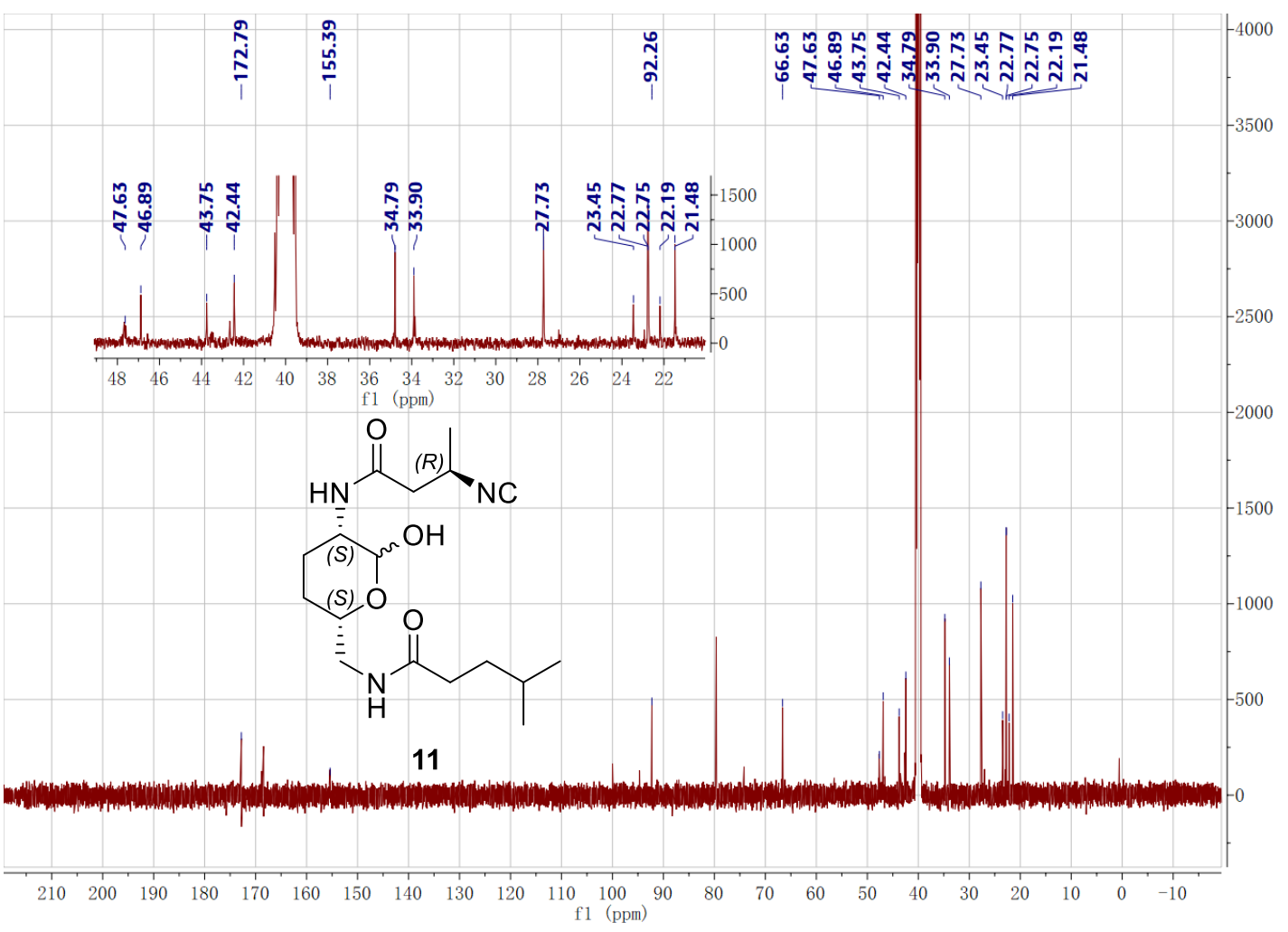


C. DEPT 90 spectrum

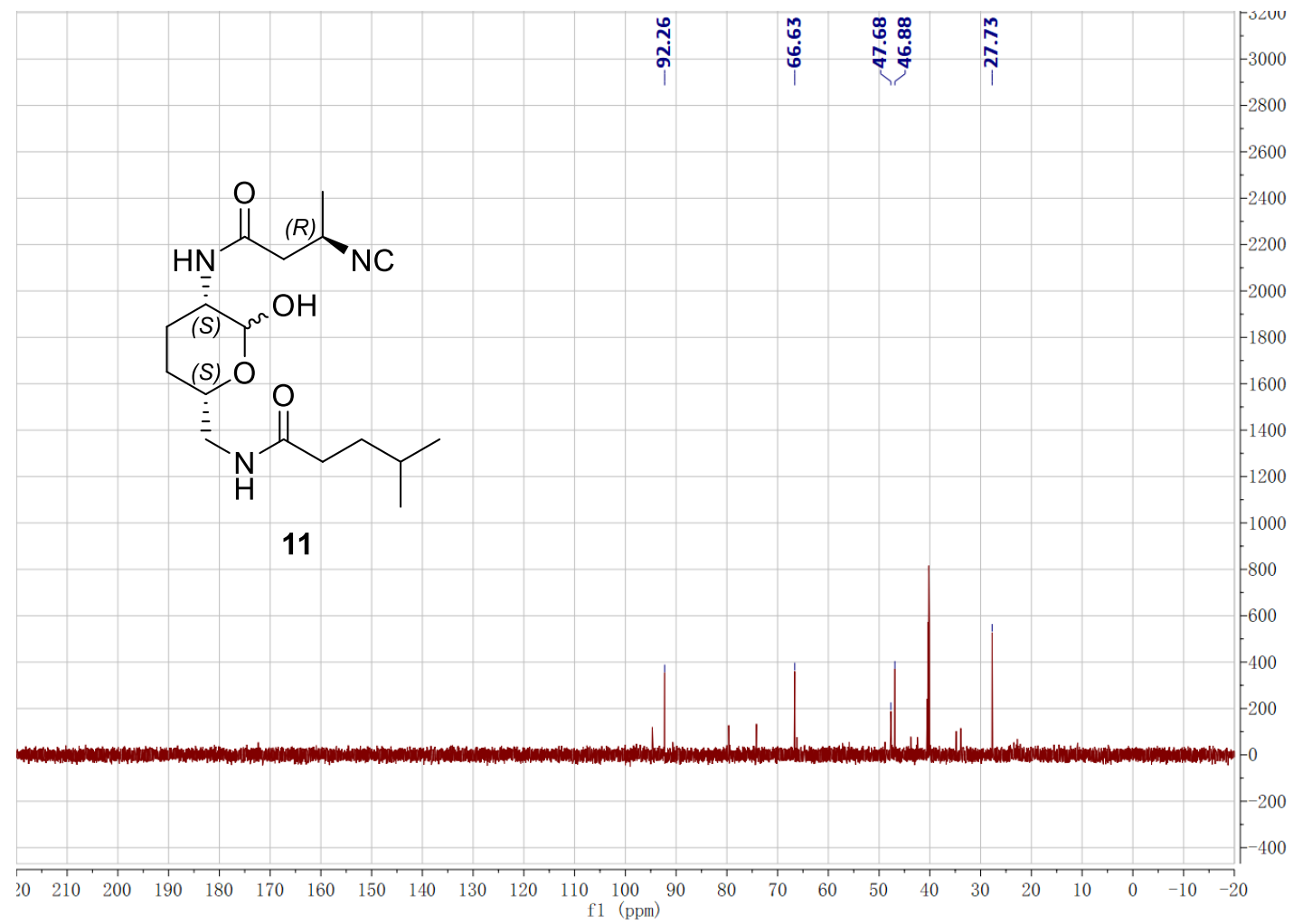

D. DEPT 135 spectrum

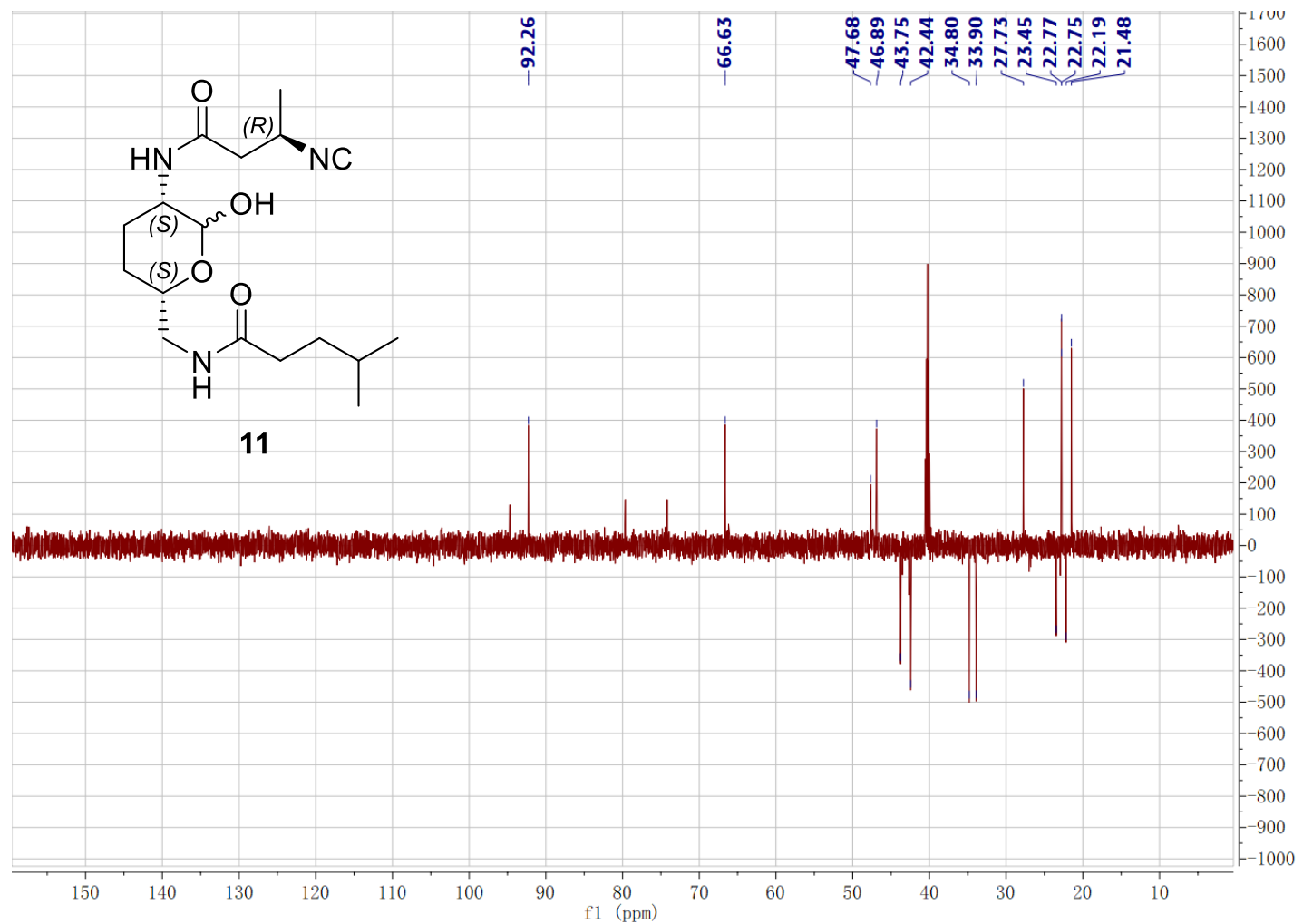


E. COSY spectrum

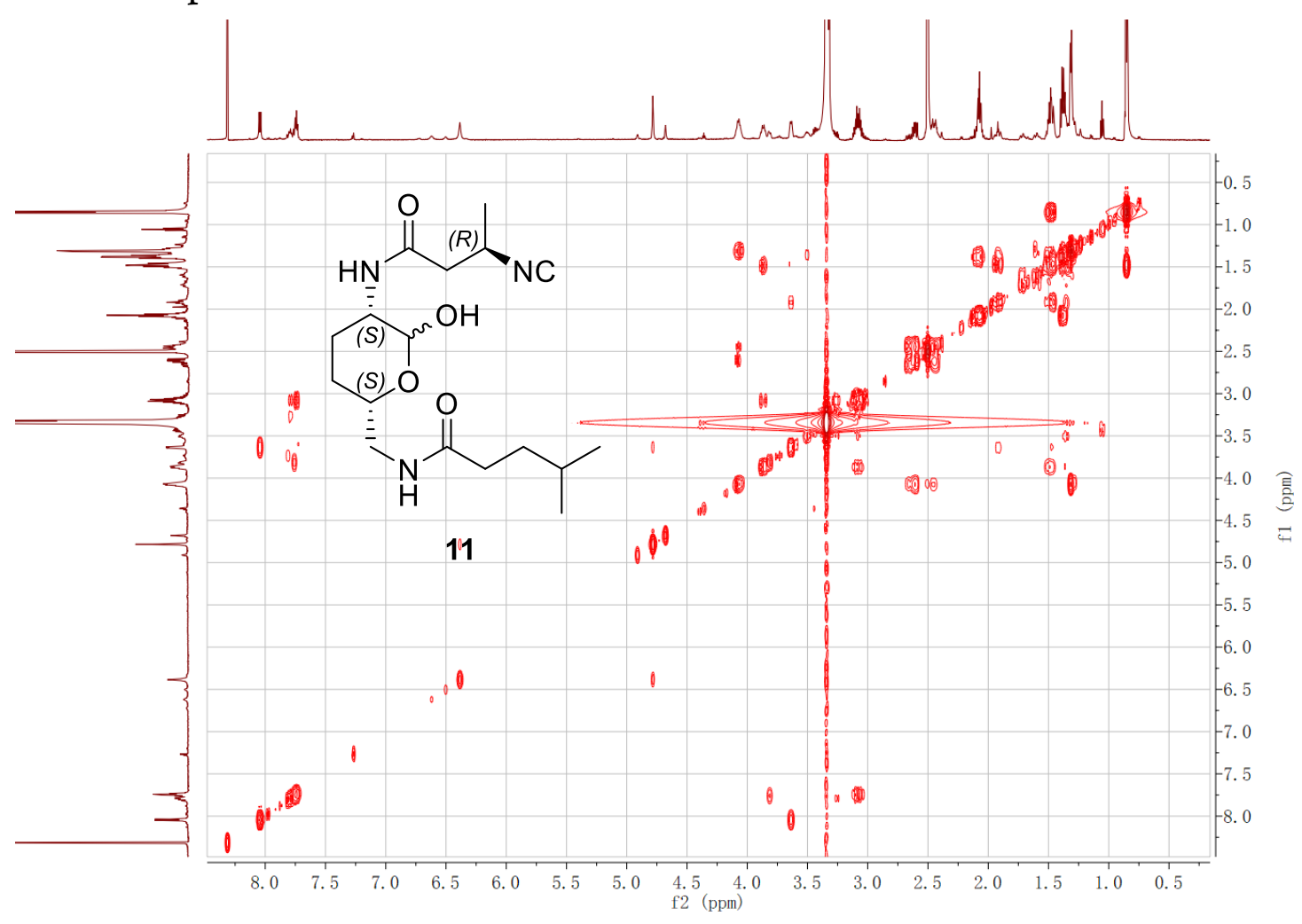

F. HSQC spectrum

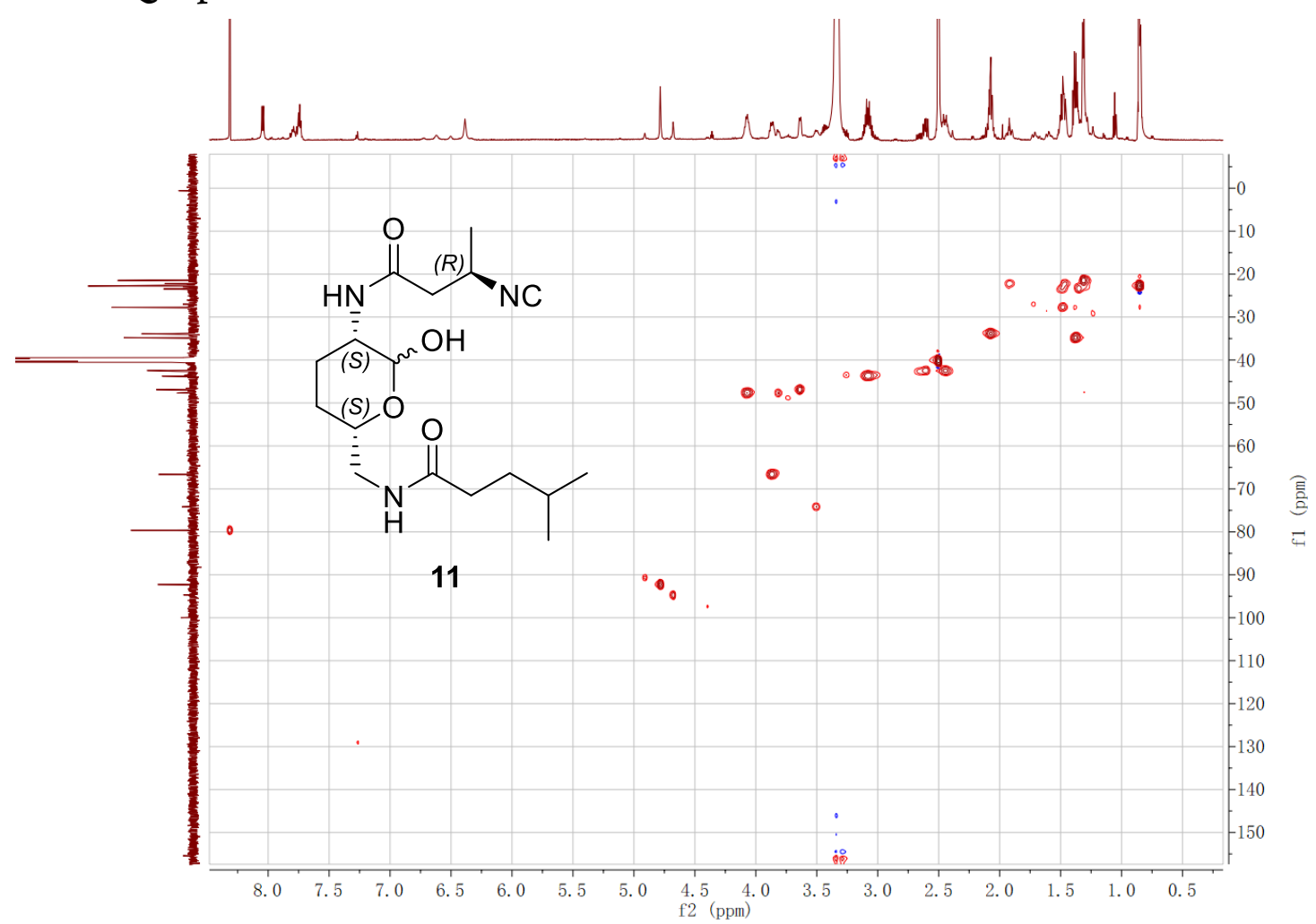


G. HMBC spectrum
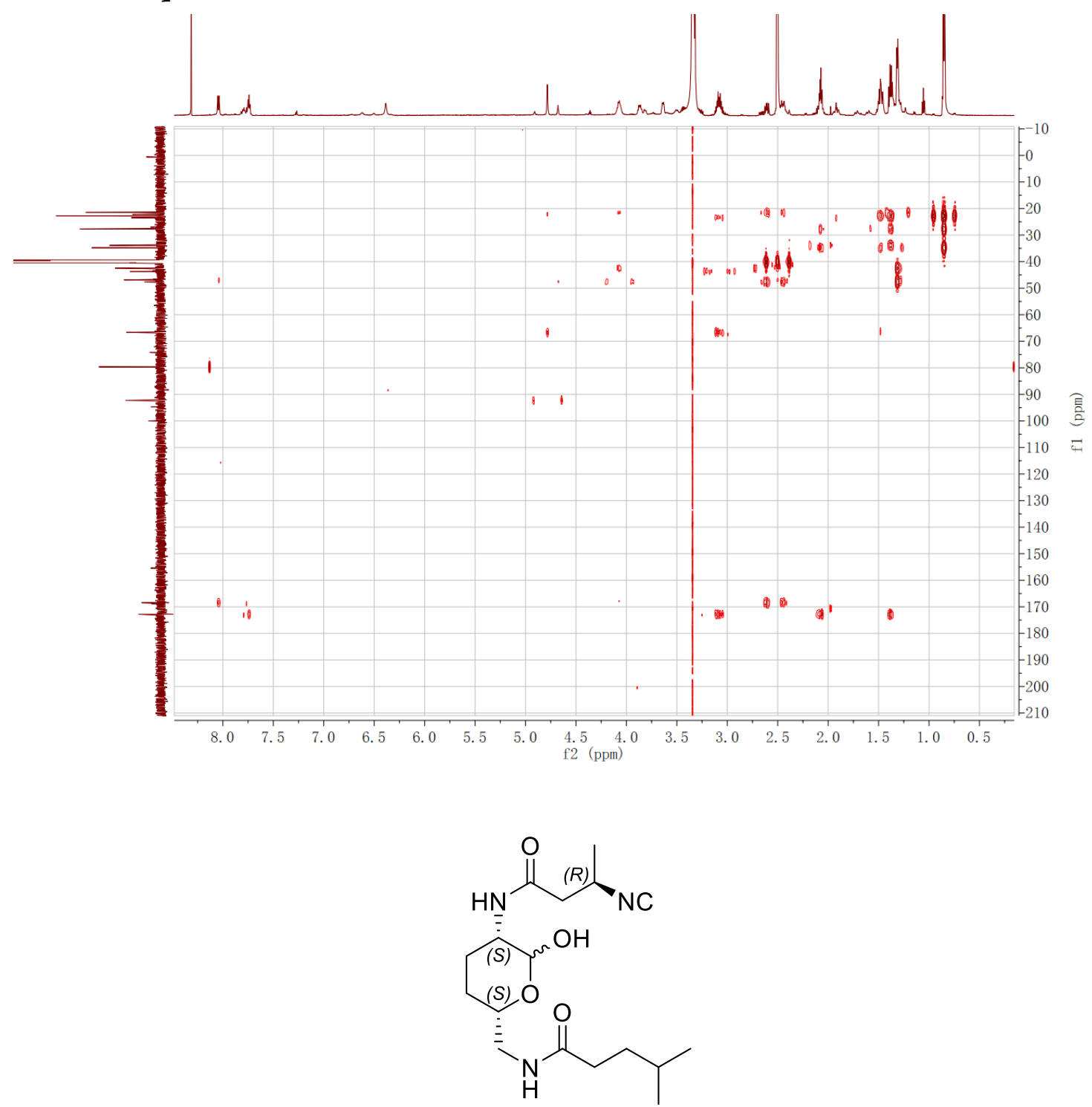

11 
Figure S7. NMR spectra of 12 in DMSO- $d_{6}$ at $600 \mathrm{MHz}$ and $151 \mathrm{MHz}$.

A. ${ }^{1} \mathrm{H}$ spectrum

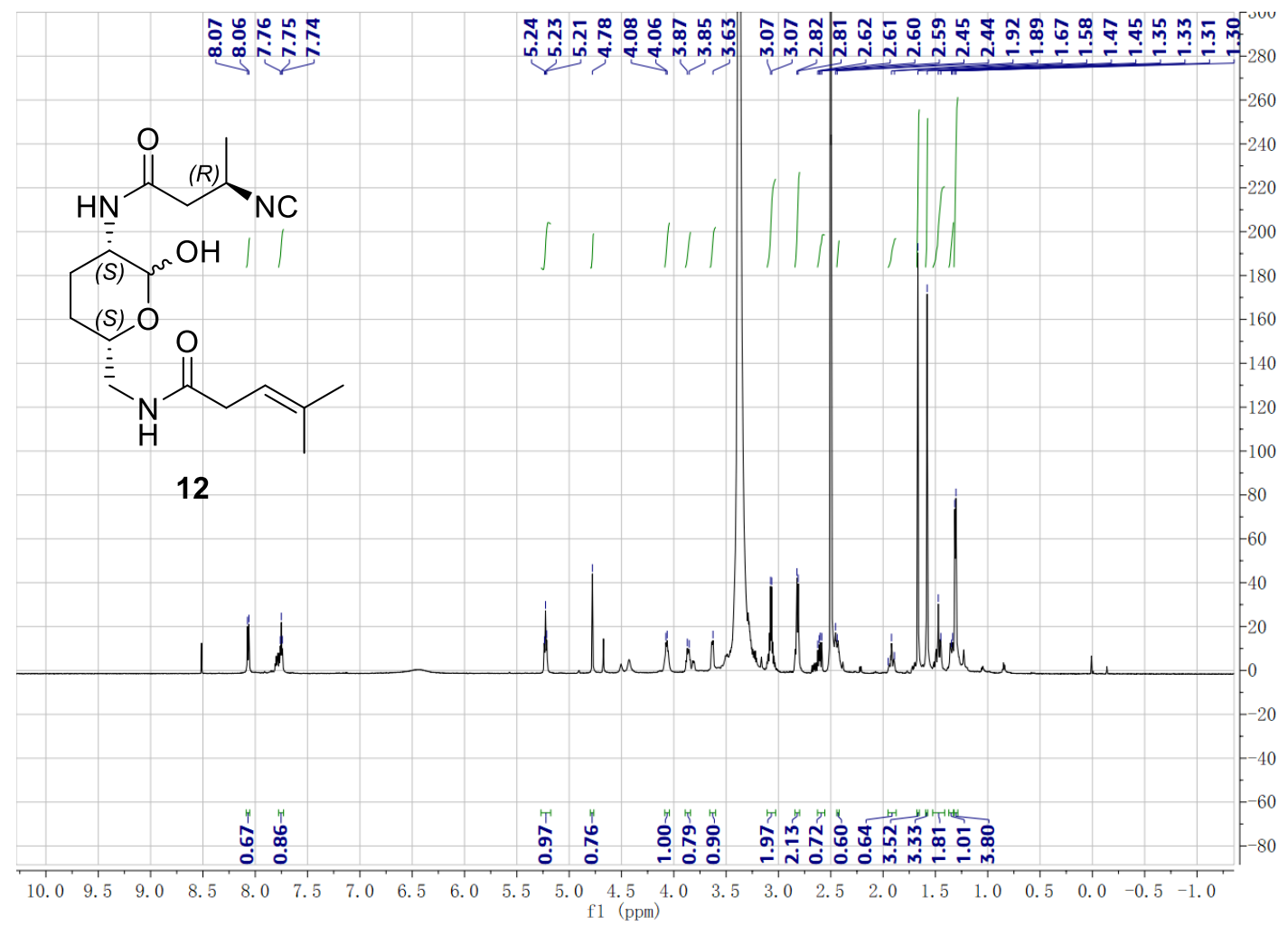

B. ${ }^{13} \mathrm{C}$ spectrum

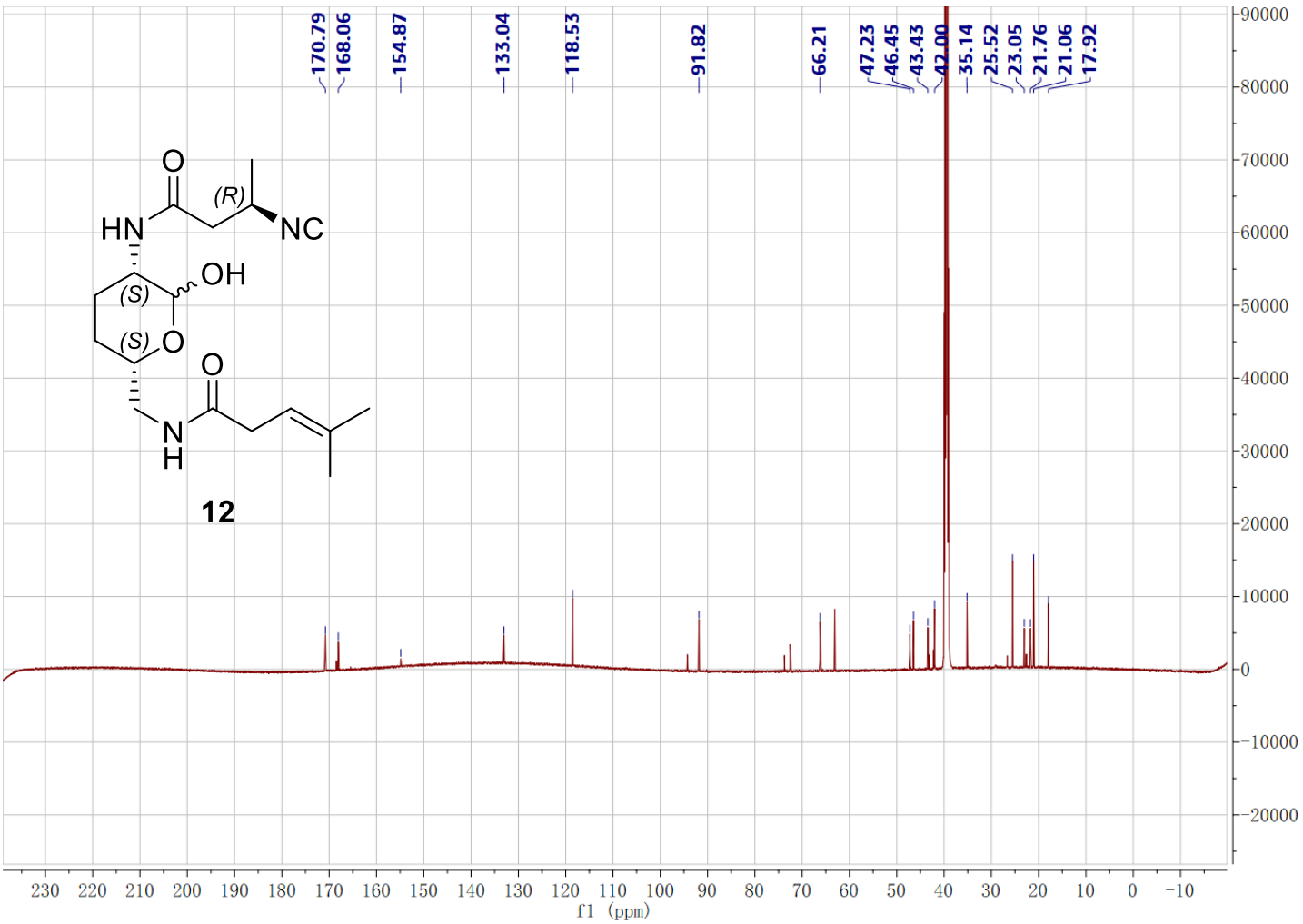


C. DEPT 90 spectrum

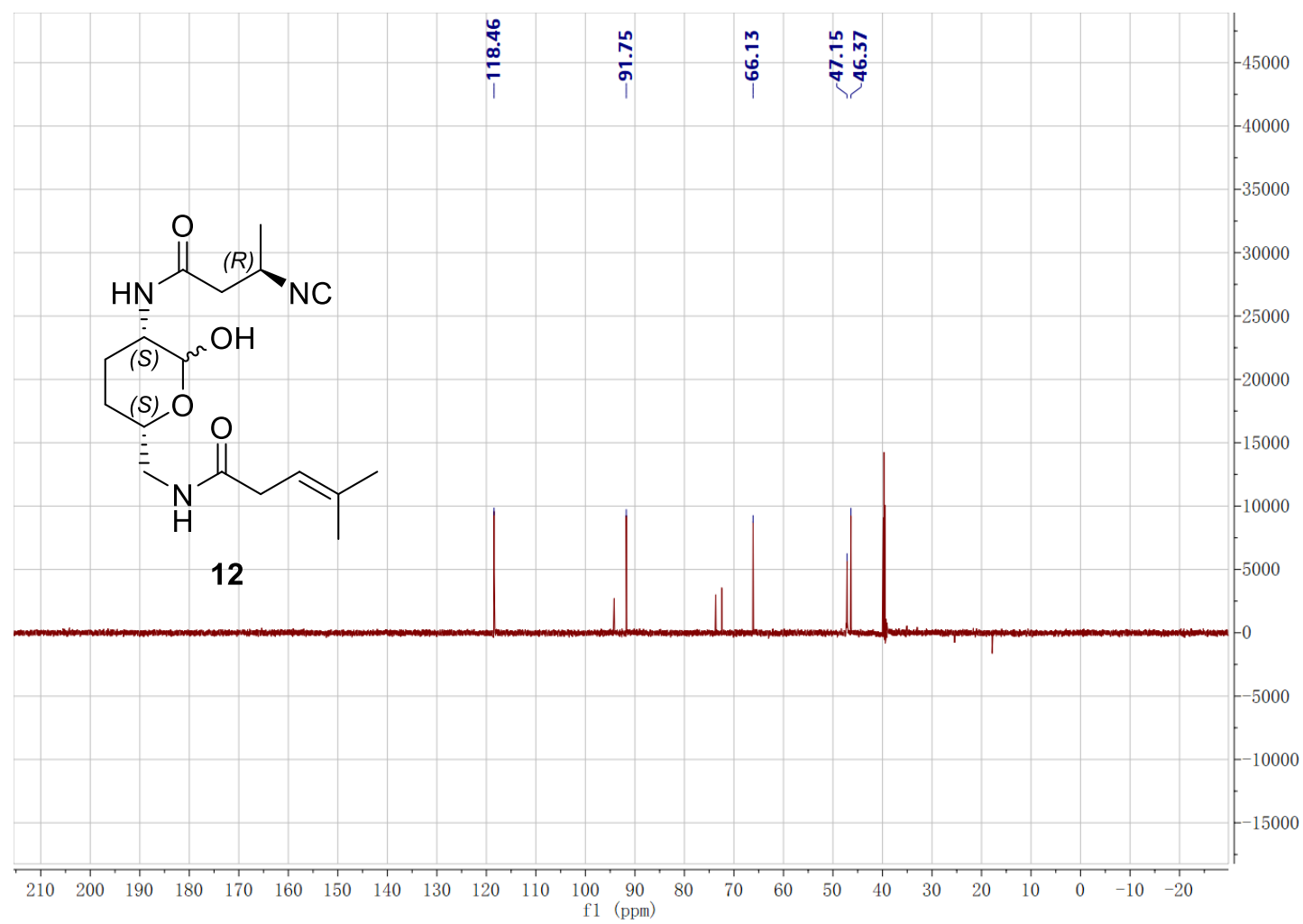

D. DEPT 135 spectrum

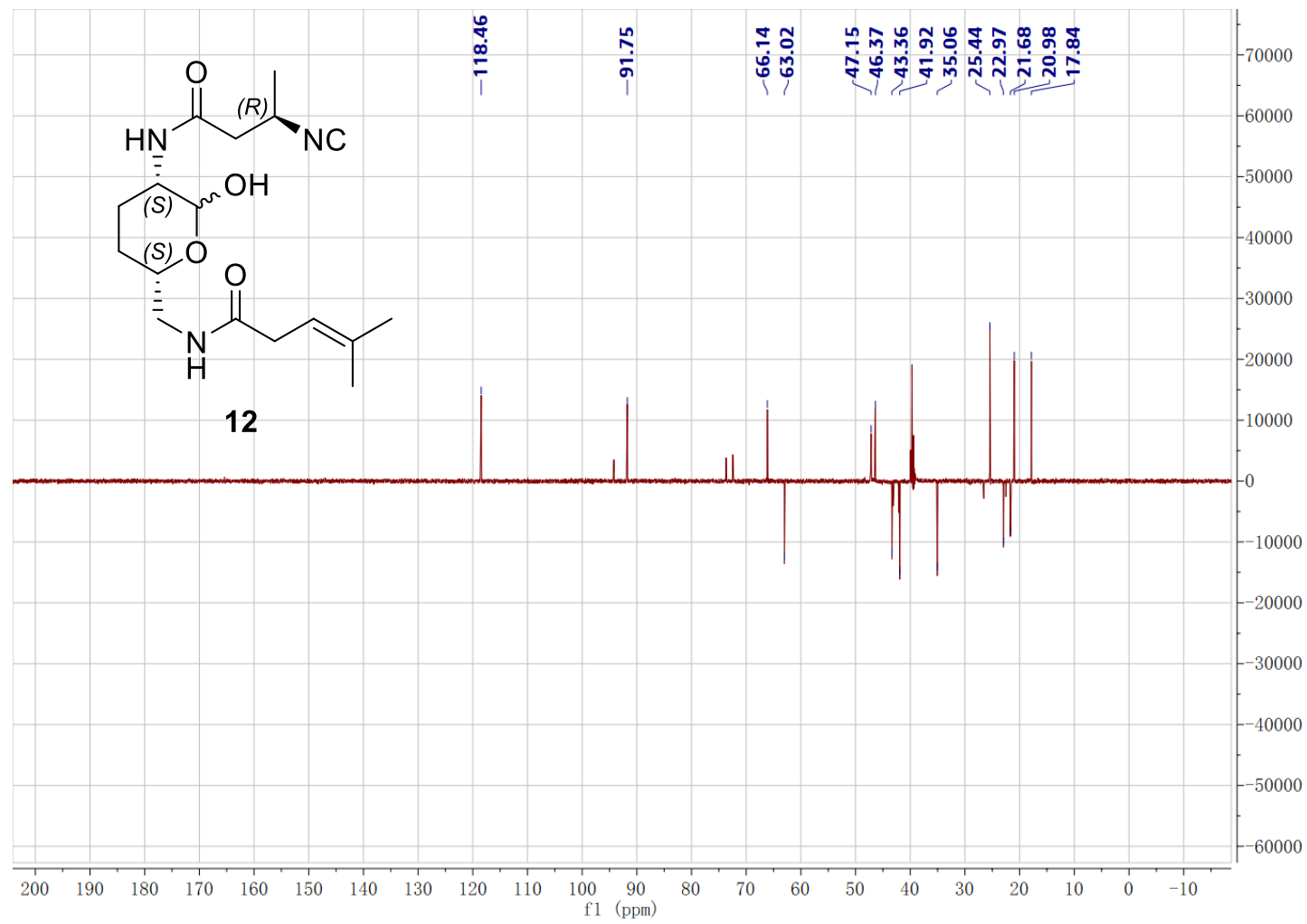


E. COSY spectrum

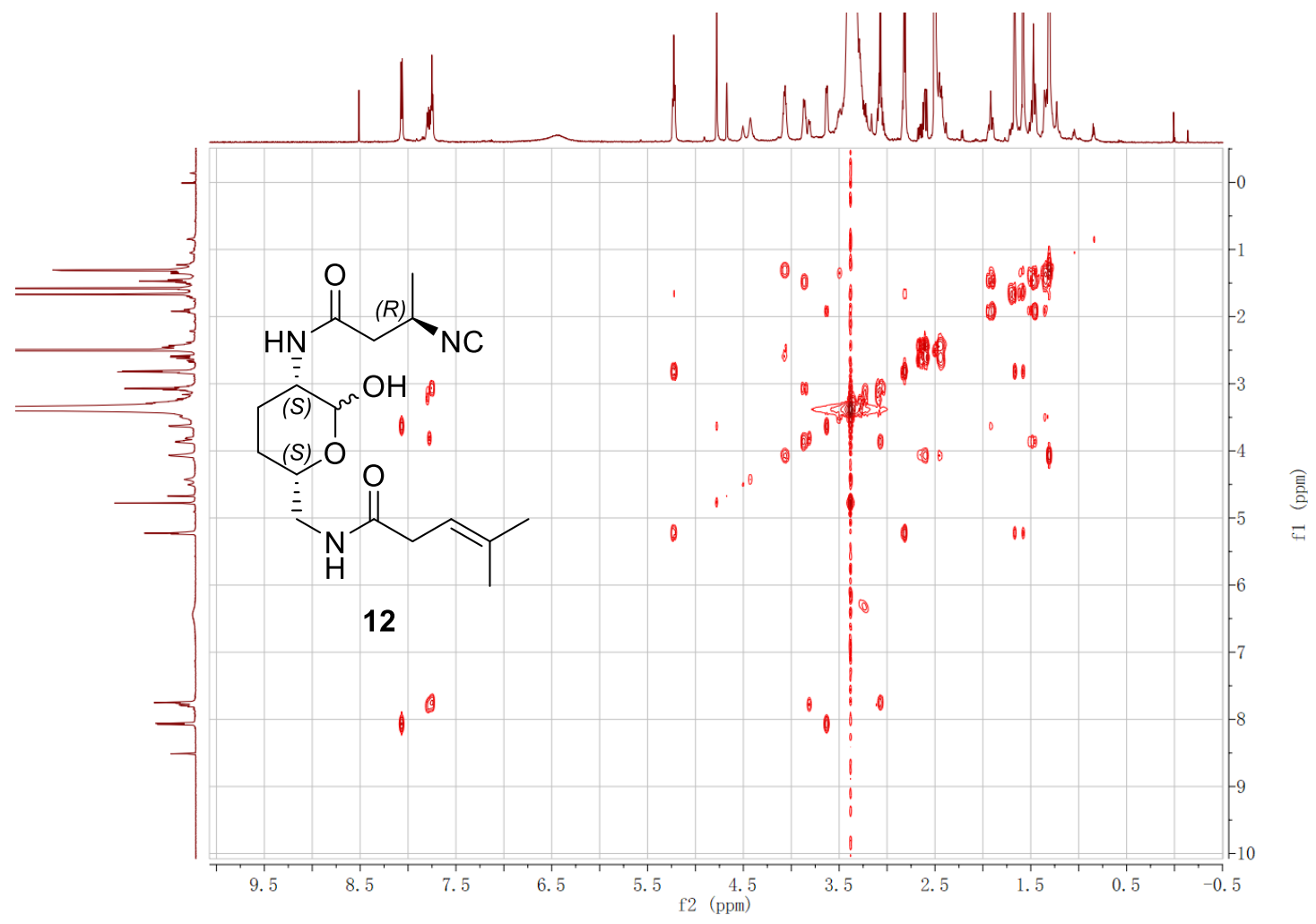

F. HSQC spectrum

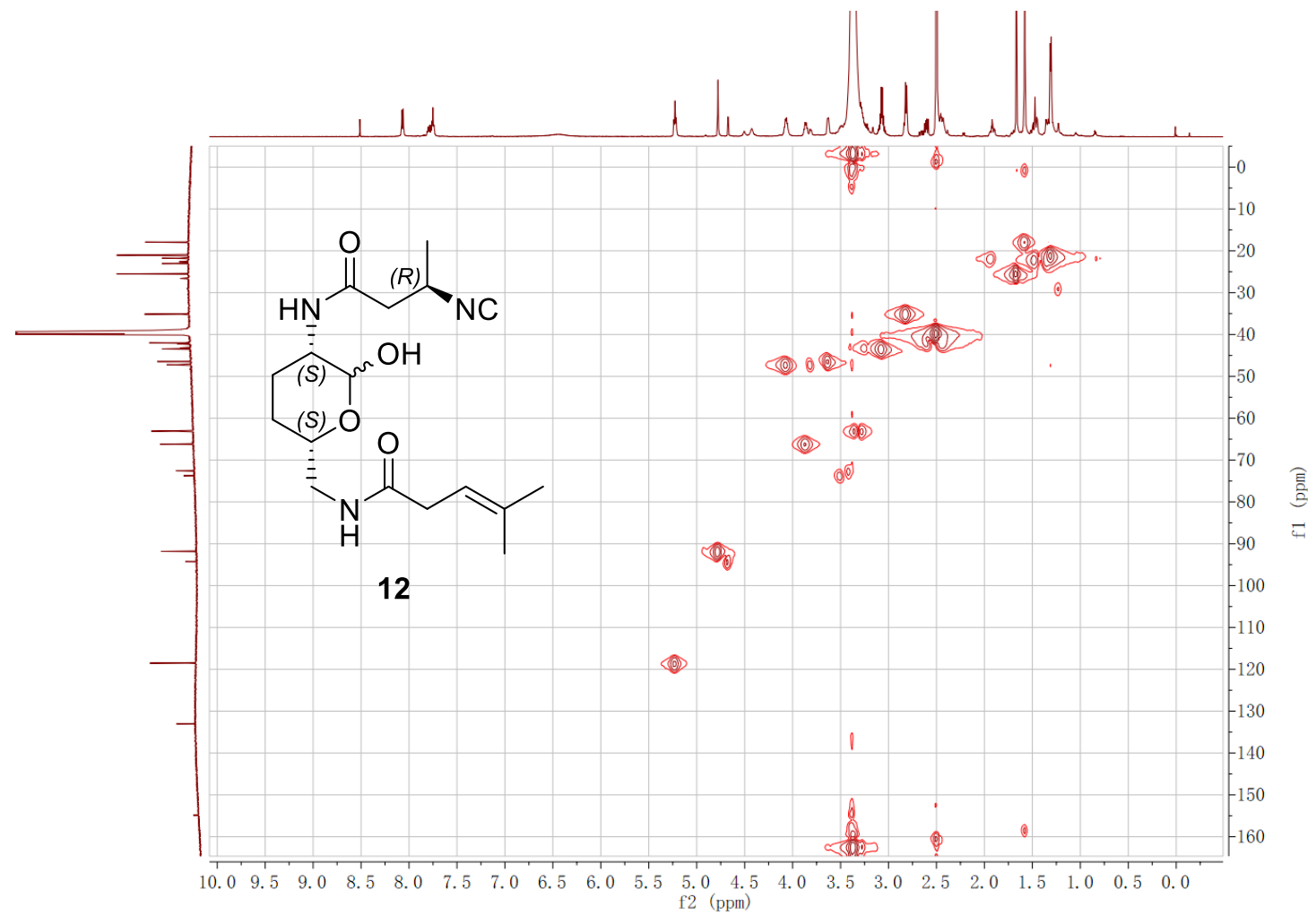


G. HMBC spectrum
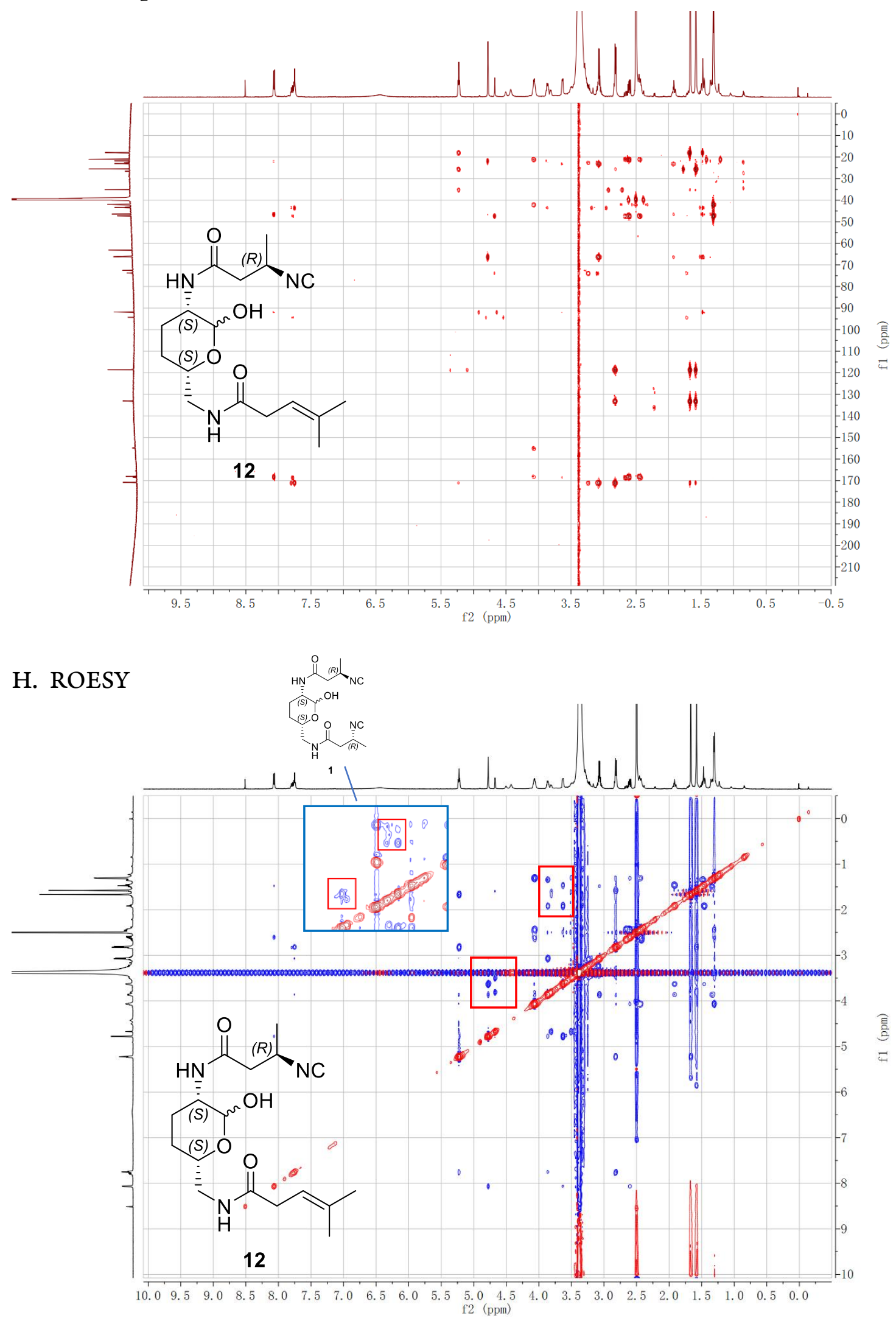